\title{
Landmines Ground-Penetrating Radar Signal Enhancement by Digital Filtering
}

\author{
Delphine Potin, Emmanuel Duflos, Member, IEEE, and Philippe Vanheeghe, Senior Member, IEEE
}

\begin{abstract}
Until now, humanitarian demining has been unable to provide a solution to the landmine removal problem. Furthermore, new low-cost methods have to be developed quickly. While much progress has been made with the introduction of new sensor types, other problems have been raised by these sensors. Ground-penetrating radars (GPRs) are key sensors for landmine detection as they are capable of detecting landmines with low metal contents. GPRs deliver so-called Bscan data, which are, roughly, vertical slice images of the ground. However, due to the high dielectric permittivity contrast at the air-ground interface, a strong response is recorded at an early time by GPRs. This response is the main component of the so-called clutter noise, and it blurs the responses of landmines buried at shallow depths. The landmine detection task is therefore quite difficult, and a preprocessing step, which aims at reducing the clutter, is often needed. In this paper, a difficult case for clutter reduction, that is, when landmines and clutter responses overlap in time, is presented. A new and simple clutter removal method based on the design of a two-dimensional digital filter, which is adapted to Bscan data, is proposed. The designed filter must reduce the clutter on Bscan data significantly while protecting the landmine responses. In order to do so, a frequency analysis of a clutter geometrical model is first led. Then, the same process is applied to a geometrical model of a signal coming from a landmine. This results in building a high-pass digital filter and determining its cutoff frequencies. Finally, simulations are presented on simulated and real data, and a comparison with the classical clutter removal algorithm is made.
\end{abstract}

Index Terms-Clutter removal, digital filtering, Fourier analysis, ground-penetrating radar (GPR), landmines.

\section{INTRODUCTION}

$\mathbf{T}$ HE MILLIONS of landmines spread out over the planet are not only a humanitarian disaster; they also hinder the social and economic development of the concerned countries. Removal of landmines, which is also called humanitarian demining, has therefore become one of the major stakes since the beginning of this century. Unfortunately, humanitarian demining faces many technical, scientific, and operational problems. For instance, landmines are small, their metallic part becomes less and less important, and they could have been laid for many years. These considerations have led scientists to the conclusion that only multisensor systems could be efficient enough to bring

Manuscript received January 18, 2005; revised February 1, 2006. This work was supported by the Fondation Norbert Ségard.

D. Potin is with the Institut Supérieur d'Électronique et du Numérique, 59046 Lille Cedex, France (e-mail: delphine.potin@isen.fr).

E. Duflos and P. Vanheeghe are with the Laboratoire d'Automatique, Génie Informatique and Signal (LAGIS), CNRS UMR 8146, Ecole Centrale de Lille, 59651 Villeneuve d'Ascq Cedex, France (e-mail: emmanuel.duflos@ec-lille.fr; philippe.vanheeghe@ec-lille.fr).

Digital Object Identifier 10.1109/TGRS.2006.875356

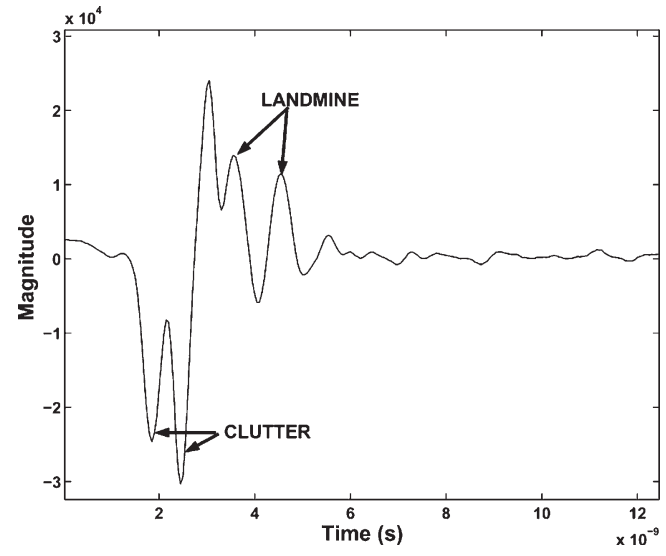

Fig. 1. Typical Ascan record obtained by a GPR pulse radar. Clutter is characterized by large amplitude oscillations at early times. This Ascan is represented by a vertical dash line on the Bscan of Fig. 2.

a solution to landmine detection problems and, therefore, to humanitarian demining. Thanks to the sensors, the technology is available. The ground-penetrating radar (GPR) has an important detection potential and has been a part of numerous multisensors systems developed these past few years. Generally speaking, in landmine detection applications, there are two kinds of GPRs, namely: 1) frequency-stepped continuous-wave (FSCW) radars and 2) pulse radars [1]. FSCW radars emit stepped radio-frequency signals toward the ground and record the response. Pulse radars emit short-duration electromagnetic (EM) pulses that propagate into the soil and reflect on the dielectric permittivity discontinuities. When recorded at a given location, the recorded pulse radar response is an Ascan, which is actually the magnitude of the reflected wave with respect to time. Due to propagation time, waves reflected on an object arrive to the GPR with a time lag, which is related ${ }^{1}$ to the distance between the object and the GPR. The image obtained by concatenating Ascans recorded along a survey line is called a Bscan. The horizontal axis of a Bscan corresponds to the GPR spatial location, ${ }^{2}$ whereas the vertical axis corresponds to time (i.e., depth). A Bscan can be seen as an image of a vertical slice of the ground. Typical Ascans and Bscans recorded in the context of landmine detection are plotted in Figs. 1 and 2.

This paper deals with pulse radar. When such radar is used for landmine detection, the emitted EM pulse strongly reflects at the air-ground interface. This results in a hindering

\footnotetext{
${ }^{1}$ The wave arrival time lag is almost proportional to the buried-object distance. The proportionality coefficient depends on the physical parameters of the soil.

${ }^{2}$ It is assumed here that the GPR is moved along a straight line. The horizontal axis actually gives the distance covered by the GPR from its initial position.
} 


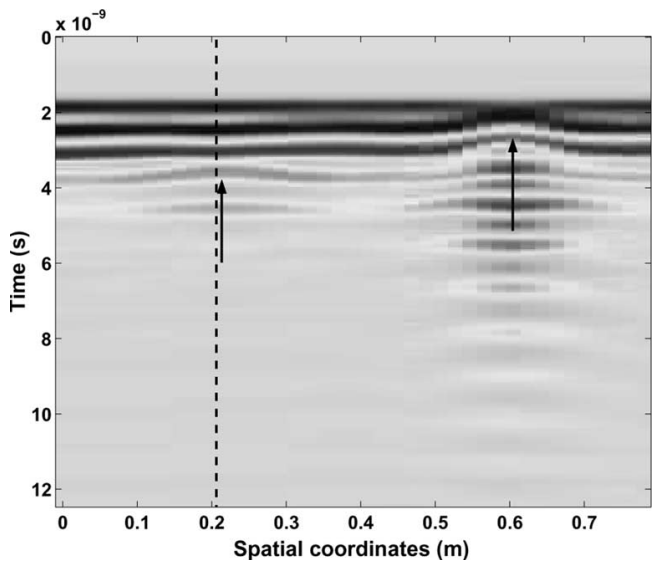

Fig. 2. Bscan image obtained by concatenating consecutive Ascans recorded along a survey line. Two typical landmine signatures (hyperbola) can be seen below the horizontal clutter stripes (arrows).

high-amplitude response, which appears at the early time of the Ascan (Fig. 1). This phenomenon is known as "clutter" and makes the detection of landmines from Ascans/Bscans difficult. More specifically, contrary of antitank mines, many landmines are actually just laid flush on the ground or buried at shallow depths $(1-5 \mathrm{~cm})$; thus, their responses to the GPR-emitted pulse overlap with clutter. Moreover, their metallic contents can be very low; their responses to the GPR-emitted wave are therefore much weaker than to the one coming from the air-ground interface. As a consequence, landmine responses are hidden in the response of the air-ground interface resulting in a poor signal-to-clutter ratio. As previously stated, clutter is mainly caused by the air-ground interface response. To a lower extent, it is also created by antenna coupling problems and multiple reflections on the air-ground interface. Therefore, one of the key problems to solve in order to improve the detection processes is clutter reduction so that the signal-toclutter ratio rises when there is a landmine or a potentially dangerous object.

Many clutter reduction methods can be summed up into two classes, which correspond to two possible signal processing approaches to increase the signal-to-clutter ratio. The first class contains methods based on classical filtering [1]-[4] in the time or frequency domains. The main method of this class is the classical clutter reduction algorithm (CCRA), which is actually a clutter processing in the time domain by a digital filter whose coefficients are not optimized with respect to the noise and signal spectra [1]. Another, but less used, approach consists of eliminating the part obtained for all instants before a given time $t_{s}$ from the signal. After $t_{s}$, the clutter is no more considered as being the predominant component of the whole signal. From a signal processing point of view, this is equivalent to windowing the signal in the time domain and therefore to filtering the spectrum as the convolution operation is done in the frequency domain. This method, which is also called "early-time gating," has a drawback, which is the elimination of responses coming from targets buried at shallow depths and whose responses would be situated at time instants of the remote time interval. Moreover, the choice of $t_{s}$ can be hard to solve [4] as the clutter is nonstationary. The second class of clutter removal processing is composed of more complex statistically based methods which model the clutter [4]-[8]. Among this class, the first method consists of removing the peak response due to the response of the air-ground interface. This peak is modeled and then subtracted to the measurements. Methods vary depending on the chosen approach to model the peak. In [5] and [6], the method is based on peak modeling by a linear combination of complex exponentials whose parameters are estimated by the Prony method. Generally speaking, these methods are based on peak modeling that result from the response of the air-ground interface and eventually from antenna coupling and the subtraction of this modeled peak from the recorded signal. However, whatever the method used, the presence of a shallow buried object modifies the early time response, which includes the clutter, and thus calls into question the validity of the model, which can be a serious drawback from an operational point of view. Van der Merwe and Gutpa [4] propose an iterative method based on the same idea which takes into account the presence of shallow buried objects and the incoherent component of the clutter, i.e., noise and nondeterministic perturbations. The main drawback of this method is that a reference signature of the buried object is needed. Other methods [7], [8] consist of using linear prediction theory to cancel the clutter. In [8], the method deals with a nonstationnary clutter environment, and the linear prediction coefficients are computed adaptively. The main drawback of this method is that it makes the assumption of Gaussian noise for the prediction error, which is not proven. In [9]-[14], clutter/signal separation techniques based on principal or independent component analysis are used. GPR data are decomposed into suitable subspace components, which make it possible to select a subset of components that contain primarily landmines and others that contain mainly clutter information. However, there is no efficient technique to select automatically which components come from clutter or a buried object, and often the selection is done by visual inspection of the components. Finally, the approach in [15] consists of studying the frequency-domain features from the GPR signal in order to improve the detection of plastic landmines. However, the preprocessing method used to remove ground bounce does not take into account the case where clutter and landmine responses overlap in time.

In this paper, we focus on a difficult case for clutter reduction, that is, when landmines and clutter responses overlap in time. A new and simple clutter removal method, based on the design of a two-dimensional (2-D) digital filter which is adapted to Bscan data, is proposed. As shown in Fig. 2, clutter appears in Bscans as three almost horizontal stripes whereas a buried object appears as a hyperbolic spreading function resulting from the imperfect directivity of the GPR antenna. The designed filter must reduce the clutter (i.e., horizontal bands) significantly on Bscan data while protecting the landmine responses (i.e., hyperbolas). Thus, detection techniques that search for hyperbolas in Bscans [16]-[18] can then be used.

This paper is organized as follows. Section II presents the real GPR data used in order to build our clutter reduction method. In Section III, a geometrical model of both clutter and landmine signatures in a Bscan is described. Then, a frequency analysis of the modeled clutter and landmine signal yields in deriving a 2-D digital filter adapted to Bscan data. Section IV presents the design of this filter. Finally, simulations results on simulated and real data are given in Section $\mathrm{V}$, and a 


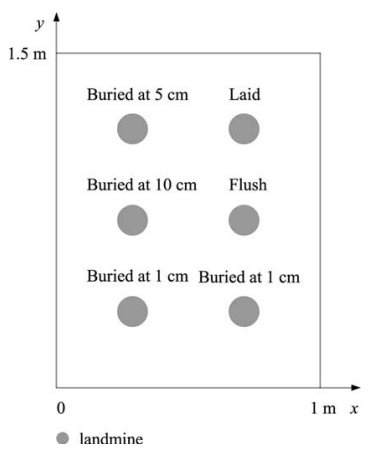

Fig. 3. Laying configurations of the mines for the different scenarios.

comparison with a classical clutter reduction method is made in Section VI in order to evaluate the performances of our method.

\section{GPR DATA}

The pulse radar used is a handheld radar originally designed for civil engineering from ERA Technology. The transmitting antenna regularly emits a short pulse of EM energy. The part of the emitted signal that is reflected and/or diffracted is recorded by the radar receiving antenna. The radar frequency, which is the central frequency of the emitted pulse spectrum, is $1 \mathrm{GHz}$. The set of real data was recorded during the Multisensor Acquisition Campaign for Analysis and Data Fusion of Antipersonnel Mines campaign of measurement. The bench allows scanning, line by line in the abscissa direction, of an area of the ground measuring $1 \times 1.5 \mathrm{~m}$ with a $2-\mathrm{cm}$ step in both directions, that is, an amount of $N_{x}=50$ by $N_{y}=75$ Ascans for each scanned area. By concatenating all the Ascans in the $x$ direction, a set of $N_{y}=75$ Bscan data is obtained. Each area contains six landmines in six different laying configurations and is called a scenario. For each scenario, the laying configurations and positions of the mines are unchanged (Fig. 3). Only the type of the landmines and the nature of the soil change. There are three types of soils (clean agricultural soil, sand, and existing soil type) and three types of landmines. The MAUS1 is a metal landmine, while the AUPS and VSMK2 are plastic landmines (with low metal contents). These mines have a cylindrical shape with similar sizes (the diameter is about $9 \mathrm{~cm}$, and the height is about $3 \mathrm{~cm}$ ). Typical Ascan and Bscan data recorded by such radar are displayed in Figs. 1 and 2.

\section{Modeling And AnAlysis of Clutter AND LANDMINE SIGNATURE}

The chosen clutter reduction method is based on the use of a 2-D digital filter that is adapted to GPR data. A comparison between the clutter- and landmine-signature-approximated spectra should be made in order to find which frequency components can be filtered out to reduce clutter without distorting the landmine signatures too much.

\section{A. Clutter Geometrical Modeling and Frequency Analysis}

In a typical Bscan (see Fig. 4), the clutter appears as three horizontal bands. These bands have a very high contrast, i.e., they can be modeled as a rectangle function. Let Bscans be

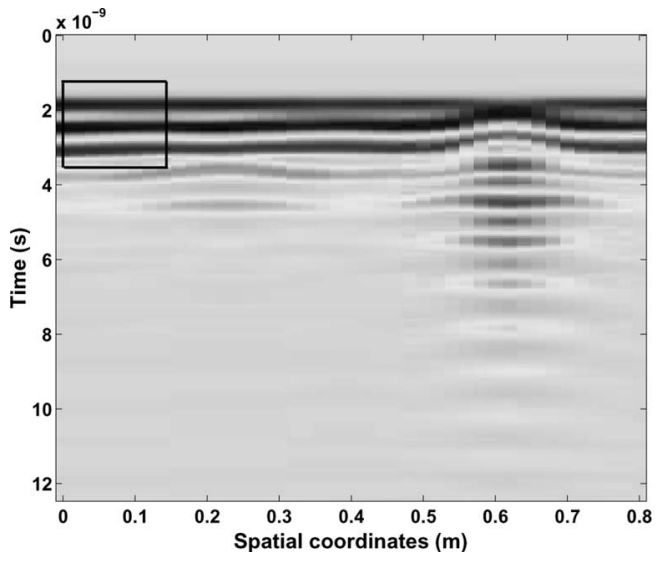

Fig. 4. Bscan of Fig. 2 before clutter reduction.

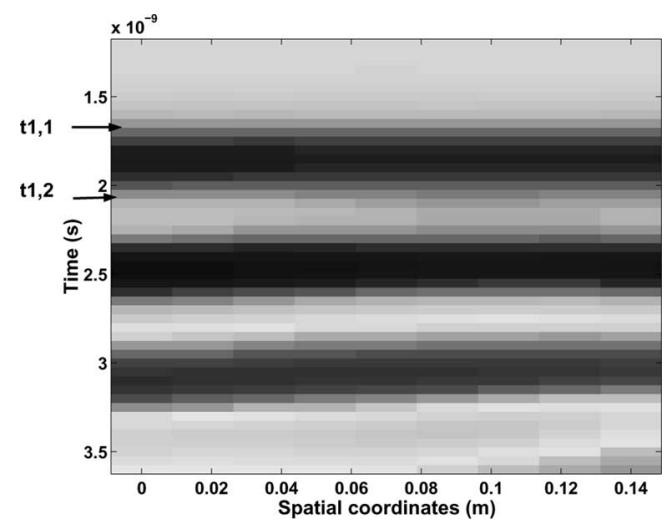

Fig. 5. Zoom of the Bscan of Fig. 4. $t_{1,1}$ and $t_{1,2}$ indicate the edges of the first clutter band.

defined as functions $I(x, t)$, where $x$ represents the spatial coordinate ranging from 0 to $x_{1}$ and $t$ represents the time coordinate ranging from 0 to $T$.

Each clutter band appearing on a Bscan can be modeled by a function $\Pi_{i}(x, t)$ defined as follows:

$$
\Pi_{i}(x, t)=\Pi_{i, 1}(x) \Pi_{i, 2}(t)
$$

with

$$
\left\{\begin{array} { l l } 
{ \Pi _ { i , 1 } ( x ) = A _ { i } } & { x \in [ 0 , x _ { 1 } ] } \\
{ \Pi _ { i , 1 } ( x ) = 0 } & { x \notin [ 0 , x _ { 1 } ] }
\end{array} \left\{\begin{array}{ll}
\Pi_{i, 2}(t)=A_{i} & t \in\left[t_{i, 1}, t_{i, 2}\right] \\
\Pi_{i, 2}(t)=0 & t \notin\left[t_{i, 1}, t_{i, 2}\right]
\end{array}\right.\right.
$$

where $A_{i}$ is the magnitude of the $i$ th clutter band and $t_{i, 1}$ and $t_{i, 2}$ are the time instants that delimit the $i$ th clutter band (Fig. 5).

Let us now consider Bscan data containing only the $i$ th clutter band, which can be modeled by the following function:

$$
I_{c}(x, t)=\Pi_{i}(x, t)
$$

In order to design a clutter removal filter, we map the data into the frequency domain. This can be done by using the following definition of the Fourier transform:

$$
I_{c}\left(\nu_{x}, \nu\right)=\int_{0}^{x_{1}} \int_{t_{i, 1}}^{t_{i, 2}} I_{c}(x, t) e^{-2 i \pi x \nu_{x}} e^{-2 i \pi \nu t} d x d t .
$$




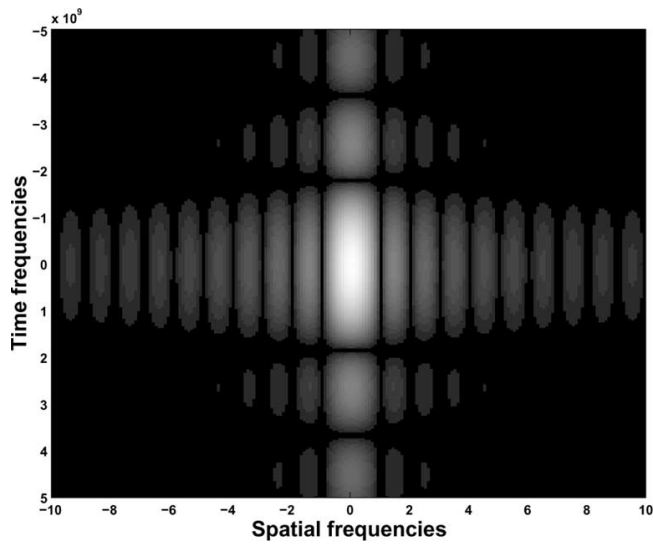

Fig. 6. Spectrum of the first clutter band represented in Figs. 4 and 5.

The derivation of $I_{c}\left(\nu_{x}, \nu\right)$ is very classical and leads to the following result:

$$
\begin{aligned}
& I_{c}\left(\nu_{x}, \nu\right)=A_{i} x_{1}\left(t_{i, 2}-t_{i, 1}\right) e^{-i \pi\left(x_{1} \nu_{x}+\left(t_{i, 1}+t_{i, 2}\right) \nu\right)} \\
& \operatorname{sinc}\left(\pi x_{1} \nu_{x}\right) \operatorname{sinc}\left(\pi\left(t_{i, 2}-t_{i, 1}\right) \nu\right) .
\end{aligned}
$$

Hence, the $i$ th clutter band has the following 2-D magnitude spectrum:

$$
\left|\Pi_{i}\left(\nu_{x}, \nu\right)\right|=A_{i} x_{1} T_{i} \operatorname{sinc}\left(\pi x_{1} \nu_{x}\right) \operatorname{sinc}\left(\pi T_{i} \nu\right)
$$

where $\nu_{x}$ is the spatial frequency parameter, $\nu$ is the frequency, and $T_{i}$ is the width of the $i$ th clutter band $\left(T_{i}=t_{i, 2}-t_{i, 1}\right)$. By considering that the main energy of such a function is located inside the first two lobes of the sinc functions, the clutter band energy is located inside the subspace $S_{c}$ defined as

$$
S_{c}=\left\{\left(\nu_{x}, \nu\right) \text { such that }\left|\nu_{x}\right| \in\left[0, \frac{2}{x_{1}}\right] \text { and }|\nu|<\frac{2}{T_{i}}\right\} .
$$

An example of such a clutter band spectrum is represented in Fig. 6 for a Bscan with the parameters $x_{1}=1 \mathrm{~m}, t_{1,1}=$ $1.510^{-9} \mathrm{~s}$, and $t_{1,2}=210^{-9} \mathrm{~s}$ (Fig. 5). As depicted in Fig. 6, the main part of the energy is well located in the subspace $S_{c}$ with

$$
S_{c}=\left\{\left(\nu_{x}, \nu\right)|| \nu_{x}|\in[0,2],| \nu \mid \in\left[0,410^{9}\right]\right\} .
$$

On a real Bscan data as the one displayed in Fig. 4, clutter appears as three horizontal bands. Hence, a clutter model for this Bscan data can be defined as follows:

$$
I_{\text {clutter }}(x, t)=\sum_{i=1}^{3} \Pi_{i}(x, t) .
$$

The total clutter spectrum is obtained by using the 2-D Fourier transform defined by (3) and is given by

$$
I_{\text {clutter }}\left(\nu_{x}, \nu\right)=\sum_{i=1}^{3} \Pi_{i}\left(\nu_{x}, \nu\right)
$$

where $\Pi_{i}\left(\nu_{x}, \nu\right)$ is the spectrum of the $i$ th clutter band.

It is then important to notice that the magnitude of the total clutter spectrum is not the sum of the magnitude of each individual clutter band spectrum. Rather, it is the magnitude of

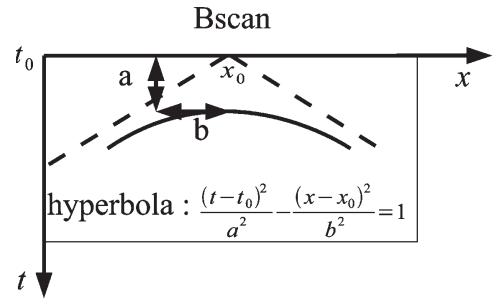

Fig. 7. Hyperbola that models the response of a buried object and its asymptote (dashed line).

the coherent sum of each individual spectrum. Having said that, the resulting spectrum cannot be wider than the spectrum of the widest individual clutter band, and thus, this widest individual spectrum provides the worst case for the purposes of selecting the cutoff frequencies of the digital filter for clutter reduction. As a consequence, to design our filter for the clutter model, we only consider the clutter band that has the widest spectrum (the smallest value for $T_{i}$ ) and whose main energy is situated inside the subspace $S_{c}\left(x_{1}, \nu_{\max }\right)$, which is defined as follows:

$$
S_{c}\left(x_{1}, \nu_{\max }\right)=\left\{\left(\nu_{x}, \nu\right) \text { such that }\left|\nu_{x}\right|<\frac{2}{x_{1}} \text { and }|\nu|<\nu_{\max }\right\}
$$

where $\nu_{\max }=2 / T_{\min }$ with $T_{\min }=\min \left(T_{i}\right), i=1, \ldots, 3$. Hence, the filter design does not depend on the number of clutter bands that appear in the Bscan.

\section{B. Landmine Signature Geometrical Modeling and Analysis}

As opposed to clutter, a buried object appears as a hyperbola in Bscans. In fact, the antenna spatial response is convolved with the target spatial response. A geometrical straightforward approach can be used in the case of a soil with known constant velocity $v$ [1]. For a pointlike object buried at depth $z_{0}$ and at position $x_{0}$, the reflected signal will be centered on a time

$$
t=t_{0}+\frac{2}{v} \sqrt{\left(x-x_{0}\right)^{2}+z_{0}^{2}}
$$

depending on the velocity $v$ of the pulse radar in the soil and the known constant time $t_{0}$ of the signal reflected from the air-ground interface. It is assumed that the distance between the GPR and the ground is kept constant during the measurement and that the air-ground interface is flat. Therefore, the equation of a hyperbola appearing in a Bscan (see Fig. 7) is

$$
\frac{\left(t-t_{0}\right)^{2}}{a^{2}}-\frac{\left(x-x_{0}\right)^{2}}{b^{2}}=1
$$

with $a=2 z_{0} / v$ and $b=z_{0}$.

Using this classical result, the modeling of a Bscan containing only a hyperbola such as (12) can be done by defining this Bscan as

$I_{s}(x, t)=\delta\left(t-t_{0}-a \sqrt{\frac{\left(x-x_{0}\right)^{2}}{b^{2}}+1}\right), \quad$ if $\left|x-x_{0}\right| \leq \Delta x$

$I_{s}(x, t)=0$,

else 


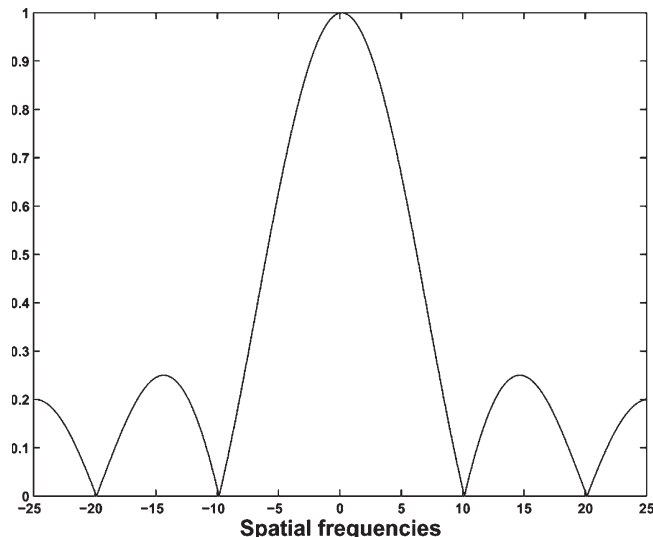

Fig. 8. Hyperbola approximated spectrum for $\left|\Delta_{x}\right|<b$ and $\nu=0$.

with $\delta(t-\alpha)$ as the Dirac function at point $t=\alpha$. The width of such a hyperbola is $2 \Delta x$. As the antenna directivity is finite, the reflected signal can only be measured from points not too far from $x_{0}$. Using definition (3) of the Fourier transform and the spectrum of the Dirac distribution, the frequency representation of a buried object can be defined as

$$
I_{s}\left(\nu_{x}, \nu\right)=e^{-2 i \pi t_{0} \nu} \int_{x_{0}-\Delta x}^{x_{0}+\Delta x} e^{-2 i \pi \nu a} \sqrt{\frac{\left(x-x_{0}\right)^{2}}{b^{2}}+1} e^{-2 i \pi x \nu_{x}} d x .
$$

Using the properties of the Fourier transform, the previous spectrum is also equal to

$$
I_{s}\left(\nu_{x}, \nu\right)=e^{-2 i \pi\left(x_{0}+t_{0}\right) \nu} \int_{-\Delta x}^{\Delta x} e^{-2 i \nu \pi a} \sqrt{\frac{x^{2}}{b^{2}}+1} e^{-2 i \pi x \nu_{x}} d x .
$$

In the case where $|x / b|$ is small in comparison to 1 , the following approximation holds:

$$
\sqrt{\frac{x^{2}}{b^{2}}+1} \simeq 1
$$

Hence, for landmines buried at depths $z_{0}$ such that $z_{0}>$ $\Delta x\left(z_{0}=b\right)$, the hyperbola spectrum can be approximated by

$$
\begin{aligned}
& I_{s}\left(\nu_{x}, \nu\right) \simeq e^{-2 i \pi \nu\left(x_{0}+t_{0}+a\right)} \int_{-\Delta x}^{\Delta x} e^{-2 i \pi \nu_{x} x} d x \\
& I_{s}\left(\nu_{x}, \nu\right) \simeq 2 \Delta x e^{-2 i \pi \nu\left(x_{0}+t_{0}+a\right)} \operatorname{sinc}\left(2 \pi \Delta x \nu_{x}\right) .
\end{aligned}
$$

An approximation of the magnitude spectrum is given as

$$
\left|I_{s}\left(\nu_{x}, \nu\right)\right| \bumpeq 2 \Delta x\left|\operatorname{sinc}\left(2 \pi \Delta x \nu_{x}\right)\right| .
$$

The magnitude spectrum is therefore almost independent of $\nu$, and the main energy is located inside the interval $\nu_{x} \in$ $[-(1 / \Delta x) ; 1 / \Delta x]$. For a hyperbola with $\Delta x=0.05 \mathrm{~cm}, \nu_{x}$ is inside the interval $[-20 ; 20]$. Fig. 8 represents the evolution of the hyperbola approximated spectrum for $\nu=0$. The spread of the widest clutter band spectrum along the $\nu_{x}$ axis is very small in comparison with the spread of the hyperbola spectrum.

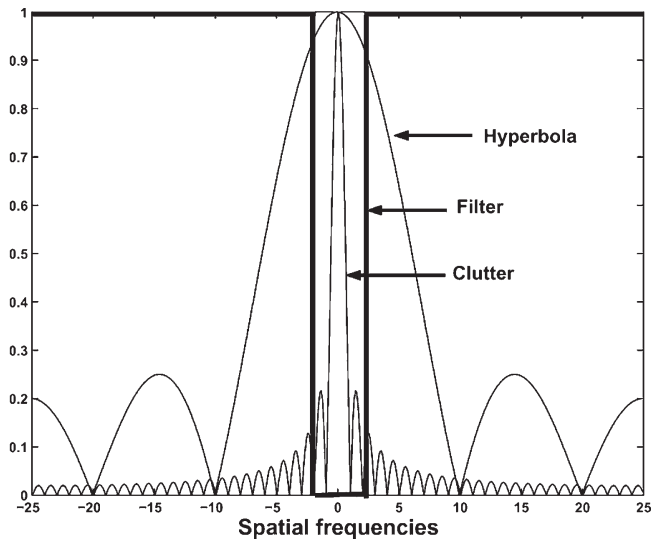

Fig. 9. Hyperbola spectrum, widest clutter band spectrum, and digital filter spectrum for $\nu=0$.

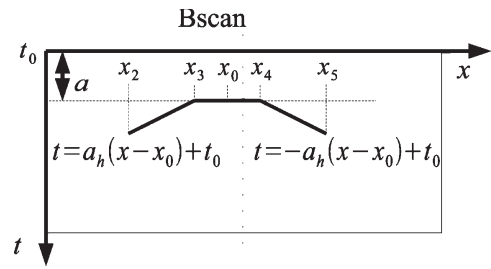

Fig. 10. Hyperbola modeled by three line segments.

Typically, the main energy is within a band of $2-\mathrm{m}^{-1}$ width for the clutter $\left(x_{1}=1 \mathrm{~m}\right)$ and within a band of $20-\mathrm{m}^{-1}$ width for the signal. Therefore, using a digital high-pass filter with a very sharp transition band to remove the clutter, as shown in Fig. 9, will not degrade the signal too much. The stopband $S$ of this filter is defined as

$$
S=\left\{\left(\nu_{x}, \nu\right) \text { such that }\left|\nu_{x}\right| \in\left[0, \frac{2}{x_{1}}\right] \quad \forall \nu\right\} .
$$

However, for landmines buried at shallow depths $(1-5 \mathrm{~cm})$, the approximation (15) does not hold for several values of $x$ and $b$ considered. It is therefore much more difficult to find an approximation of the hyperbola spectrum defined by (14). By looking at several real Bscans from our real database, such as the one displayed in Fig. 2, it can be seen that the curvature of the hyperbola top is slight. Furthermore, with the antenna directivity being finite, one can see that the hyperbola branches are of short lengths and almost symmetric. A hyperbola on a Bscan can therefore be approximated by three line segments. The hyperbola top is modeled by a line segment of null slope whose width is directly linked to the size of the buried object. The hyperbola branches are modeled by two symmetric line segments of slope $\pm a_{h}$ (see Fig. 10). A Bscan containing only one hyperbola such as the one displayed in Fig. 7 can be modeled approximately by the function $I_{m}(x, t)$ plotted in Fig. 10 and defined as

$$
\begin{aligned}
I_{m}(x, t) & =\delta\left(t=a_{h}\left(x-x_{0}\right)+t_{0}\right), & & \text { if } x \in\left[x_{2}, x_{3}\right] \\
& =\delta(t=a), & & \text { if } x \in\left[x_{3}, x_{4}\right] \\
& =\delta\left(t=-a_{h}\left(x-x_{0}\right)+t_{0}\right), & & \text { if } x \in\left[x_{4}, x_{5}\right] \\
& =0, & & \text { else }
\end{aligned}
$$




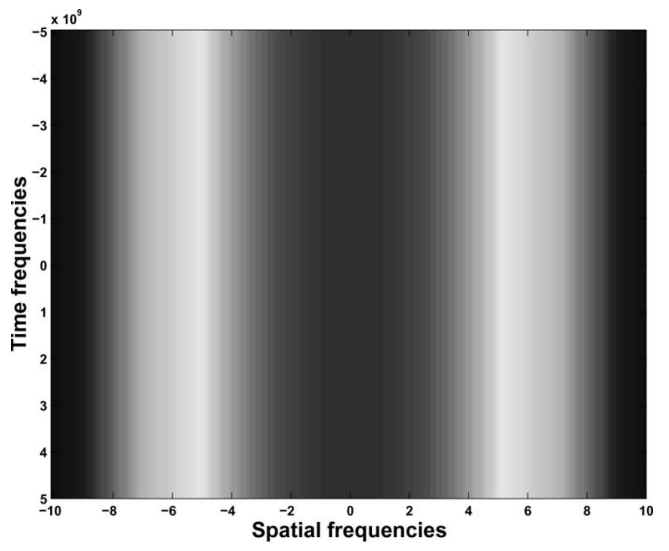

Fig. 11. Magnitude spectrum of the line segment modeling the hyperbola top.

with $a_{h}, x_{0}, x_{2}, x_{3}, x_{4}$, and $x_{5}$ being real constants. The Fourier transform $I_{m}\left(\nu_{x}, \nu\right)$ can be written as the sum of the Fourier transform of each line segment

$$
I_{m}\left(\nu_{x}, \nu\right)=I_{m_{1}}\left(\nu_{x}, \nu\right)+I_{m_{2}}\left(\nu_{x}, \nu\right)+I_{m_{3}}\left(\nu_{x}, \nu\right)
$$

with $I_{m_{1}}\left(\nu_{x}, \nu\right), I_{m_{2}}\left(\nu_{x}, \nu\right)$, and $I_{m_{3}}\left(\nu_{x}, \nu\right)$ as the Fourier transform of the line segments of slopes $a_{h}, 0$, and $-a_{h}$, respectively. An analysis of each line segment spectrum is now led in order to see which frequency components of these spectra must be kept in order to avoid landmine signature distortions by digital filtering.

The line segment modeling the top of the hyperbola defined by (21) has the following 2-D magnitude spectrum:

$$
\left|I_{m_{2}}\left(\nu_{x}, \nu\right)\right|=\left|\Delta_{t} \operatorname{sinc}\left(\pi \nu_{x} \Delta_{t}\right)\right|
$$

with $\Delta_{t}=x_{4}-x_{3}$. The main energy of such a function is located inside the subspace $S_{2}$, which is defined as follows:

$$
S_{2}=\left\{\left(\nu_{x}, \nu\right) \text { such that }\left|\nu_{x}\right| \in\left[0, \frac{2}{\Delta_{t}}\right] \quad \forall \nu\right\} \text {. }
$$

The width of the hyperbola top being linked to the size of the buried object in the landmine detection framework $\left|\Delta_{t}\right|$ can be taken around $10 \mathrm{~cm}$. Hence, as depicted in Fig. 11, the main part of the energy is located in the subspace $S_{2}=$ $\left\{\left(\nu_{x}, \nu\right)\right.$ such that $\left.\left|\nu_{x}\right| \in[0,20] \forall \nu\right\}$. As the magnitude spectrum of the hyperbola top is independent of $\nu$, a comparison between the widest clutter band spectrum and top hyperbola spectrum can be made at $\nu=0$. It is shown in Fig. 12 that the spread of the clutter spectrum along the $\nu_{x}$ axis is ten times smaller than the spread of the hyperbola spectrum. Hence, a high-pass filter designed such that the clutter situated inside $S$ is filtered out can be used because it should not bring significant distortions to the hyperbola top.

The line segment of slope $a_{h}$ modeling a hyperbola branch, defined by (20), has the following 2-D magnitude spectrum:

$$
\left|I_{m_{1}}\left(\nu_{x}, \nu\right)\right|=\left|\Delta_{b} \operatorname{sinc}\left(\pi\left(a_{h} \nu+\nu_{x}\right) \Delta_{b}\right)\right|
$$

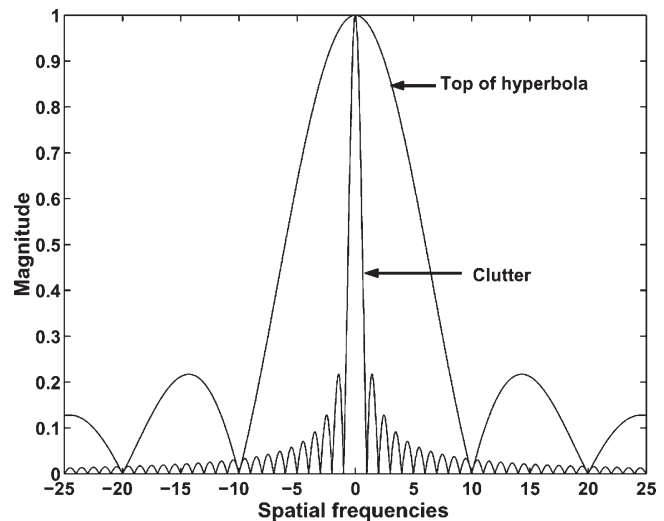

Fig. 12. Normalized magnitude spectra of the widest clutter band and of the segment line modeling the hyperbola top for $\nu=0$.

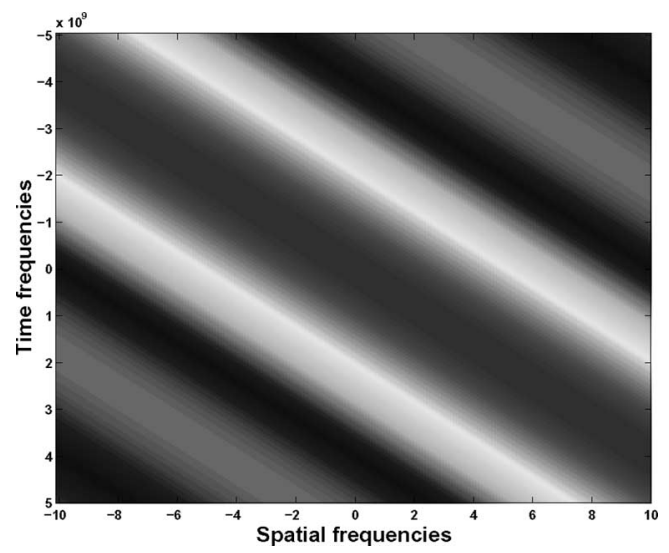

Fig. 13. Magnitude spectrum of the line segment of slope $a_{h}$.

with $\Delta_{b}=x_{3}-x_{2}$, whereas the line segment of slope $-a_{h}$ modeling the other hyperbola branch, defined by (22), has the following 2-D magnitude spectrum:

$$
\left|I_{m_{3}}\left(\nu_{x}, \nu\right)\right|=\left|\Delta_{b} \operatorname{sinc}\left(\pi\left(-a_{h} \nu+\nu_{x}\right) \Delta_{b}\right)\right|
$$

with $\Delta_{b}=x_{5}-x_{4}$ (it is supposed that the two hyperbola branches have the same length). The main energy of $\left|I_{m_{1}}\left(\nu_{x}, \nu\right)\right|$ and $\left|I_{m_{3}}\left(\nu_{x}, \nu\right)\right|$ is situated inside the first two lobes of the sinc functions. Due to the finite antenna directivity, $\Delta_{b}$ is in the range of 8-12 cm for our real Bscan database. Their slope depends on the speed at which the EM wave propagates into the soil. The magnitude spectra of the two line segments modeling the hyperbola branches, with parameters such that $\left|\Delta_{b}\right|=10 \mathrm{~cm}$ and $\left|a_{h}\right|=1$, are represented in Figs. 13 and 14 .

It is then important to notice that the intersection between the widest clutter band magnitude spectrum and hyperbola branches magnitude spectra is maximum for $\nu=0$ (see Figs. 15 and 16). The spread of the hyperbola branches magnitude spectrum is much wider than the spread of the clutter spectrum. Therefore, the frequency components of the Bscan spectrum situated inside $S$ can be filtered out without bringing many distortions to the hyperbola branches.

The analysis developed in this paragraph shows that the use of a digital high-pass filter with a very sharp transition band, whose stopband $S$ is defined by (19), must reduce significantly 


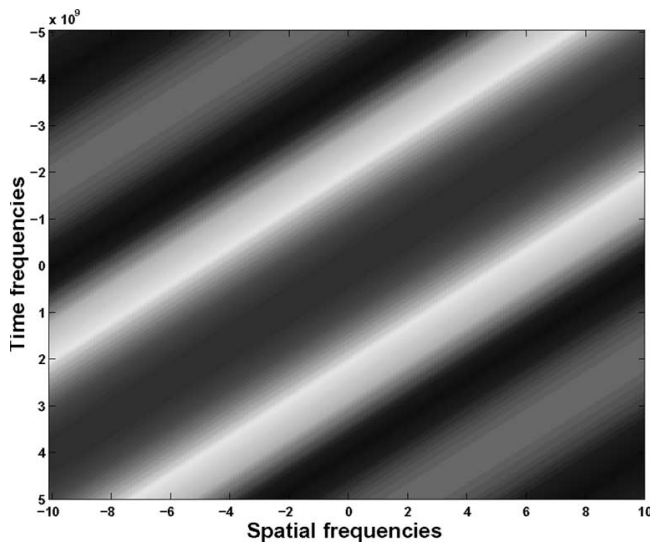

Fig. 14. Magnitude spectrum of the line segment of slope $-a_{h}$.

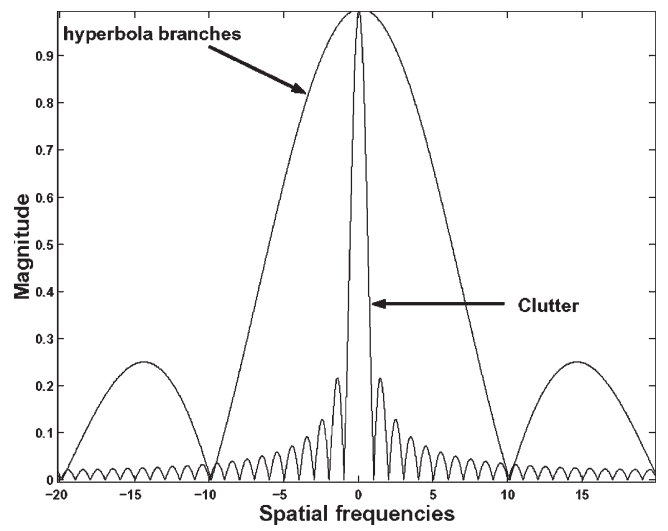

Fig. 15. Normalized magnitude spectra of the widest clutter band and of the segment lines modeling the hyperbola branches for $\nu=0$.

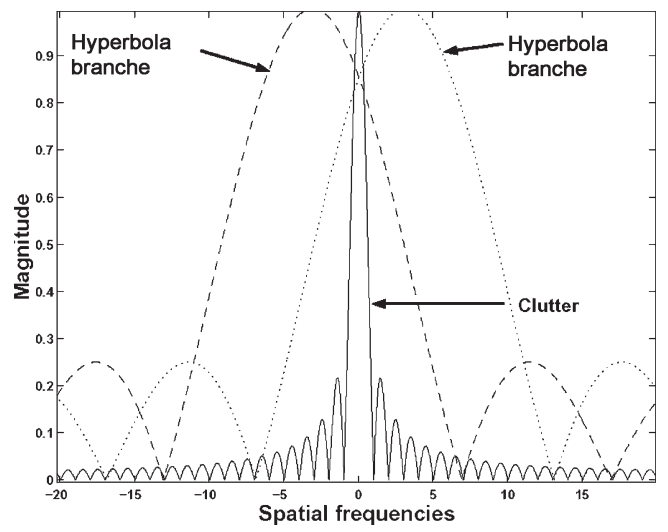

Fig. 16. Normalized magnitude spectra of the widest clutter band and of the segment lines modeling the hyperbola branches for $\nu \neq 0$.

the clutter while protecting the landmine signatures on Bscans. Indeed, whatever the depth at which a landmine is buried, the important thing to check is that the width of the landmine signature appearing on a Bscan is small in comparison with the width $x_{1}$ of this Bscan. This means that the spread of the clutter spectrum along $\nu_{x}$ will be small in comparison with the spread of a landmine spectrum. In the next section, the design and implementation of such a filter is given.

\section{Clutter Reduction by Digital Filtering}

\section{A. Specification of the Filter in the Frequency Domain}

The modeling and analysis developed in the previous section can be used to define the bandwidth of a denoising filter. The gain of the ideal filter should be equal to zero inside the interval $S$ defined by (19) and equal to one outside. Defining $H\left(\nu_{x}, \nu\right)$ as the frequency response of this ideal filter, it is expressed as

$$
\begin{cases}H\left(\nu_{x}, \nu\right)=0, & \left(\nu_{x}, \nu\right) \in S \\ H\left(\nu_{x}, \nu\right)=1, & \left(\nu_{x}, \nu\right) \notin S\end{cases}
$$

It can be noticed that because the spectrum of a landmine is almost independent of $\nu$ (Section III-B), the filter has been designed in order to filter out the clutter spectrum for all $\left(\nu_{x}, \nu\right)$ such that $\left|\nu_{x}\right| \in\left[0,2 / x_{1}\right] \forall \nu$. As a consequence, there is no cutoff frequency along the $\nu$ axis, and the filter does not depend on the time instants of the widest clutter band $t_{i, 1}$ and $t_{i, 2}$.

By taking the inverse Fourier transform, the impulse response of this filter $h(x, t)$ can be easily computed. However, the acquired image is sampled on both $x$ and $t$. Before going on, it is necessary to define an equivalent digital filter. This is classically done by using a bilinear transformation [19]. Thus, the ideal frequency response $H_{d}\left(\nu_{\mathrm{xn}}, \nu_{n}\right)$ of the digital denoising filter is defined as

$$
\begin{cases}H_{d}\left(\nu_{\mathrm{xn}}, \nu_{n}\right)=0, & \left(\nu_{\mathrm{xn}}, \nu_{n}\right) \in S_{d} \\ H_{d}\left(\nu_{\mathrm{xn}}, \nu_{n}\right)=1, & \left(\nu_{\mathrm{xn}}, \nu_{n}\right) \notin S_{d}\end{cases}
$$

where $\nu_{\mathrm{xn}}$ is the digital spatial frequency such that $\left|\nu_{\mathrm{xn}}\right| \in$ $\left[0, \nu_{\mathrm{xs}} / 2\right]$ with $\nu_{\mathrm{xs}}$ being the sampling frequency in $x$ and $\nu_{n}$ is the digital time frequency such that $\left(\left|\nu_{n}\right| \in\left[0, \nu_{s} / 2\right]\right)$ with $\nu_{s}$ being the sampling frequency in $t . S(d)$ is defined as

$$
S_{d}=\left\{\left(\nu_{\mathrm{xn}}, \nu_{n}\right) \text { such that }\left|\nu_{\mathrm{xn}}\right| \in\left[0, \alpha_{x}\right] \text { and }\left|\nu_{n}\right| \in\left[0, \frac{\nu_{s}}{2}\right]\right\}
$$

where $\alpha_{x}$, the cutoff frequency along $\nu_{x}$, is given as

$$
\alpha_{x}=\frac{\nu_{\mathrm{xs}}}{\pi} \arctan \left(\frac{2 \pi}{x_{1} \nu_{\mathrm{xs}}}\right) .
$$

\section{B. Derivation of the Impulse Response}

Using the inverse discrete Fourier transform, it is possible to derive from (30) the impulse response $h(m, n)$ of the digital filter as

$$
h(m, n)=\delta(m, n)-\frac{2 \alpha_{x}}{\nu_{\mathrm{xs}}} \operatorname{sinc}\left(\frac{2 \pi m \alpha_{x}}{\nu_{\mathrm{xs}}}\right) \operatorname{sinc}(\pi n) .
$$

Of course, this impulse response is infinite and must be truncated so that it can be implemented. The preceding expression is multiplied by a hanning window, denoted as $w(m, n)$, resulting in the following final impulse response $h_{f}(m, n)$ :

$$
h_{f}(m, n)=h(m, n) \times w(m, n)
$$


for $m \in[0, M-1]$ and $n \in[0, N-1]$ with

$$
w(m, n)=\frac{\left(1-\cos \left(\frac{2 \pi m}{M-1}\right)\right)\left(1-\cos \left(\frac{2 \pi n}{N-1}\right)\right)}{4}
$$

where $M$ and $N$ are the space and time samples numbers of the hanning window, respectively. Therefore, the real frequency response, denoted as $H_{\mathrm{df}}\left(\nu_{\mathrm{xn}}, \nu_{n}\right)$, is the convolution between the ideal frequency response and the spectrum of the 2-D hanning window, denoted as $W\left(\nu_{\mathrm{xn}}, \nu_{n}\right)$, and is expressed as

$$
H_{\mathrm{df}}\left(\nu_{\mathrm{xn}}, \nu_{n}\right)=H_{d}\left(\nu_{\mathrm{xn}}, \nu_{n}\right) * W\left(\nu_{\mathrm{xn}}, \nu_{n}\right) .
$$

\section{Tuning the Filter Parameters}

The filter parameters are $x_{1}$ and the numbers of filter coefficients $M$ and $N$. Indeed, $M$ and $N$ are the space and time samples numbers, respectively, of the hanning window used to truncate the impulse response (35).

The parameter $x_{1}$ corresponds to the distance along which the Ascans forming the Bscan data are collected by the GPR. The real filter is obtained by the convolution in the frequency domain of the ideal filter frequency response with the hanning window frequency response (36). This convolution product should be null inside the subspace $S_{d}$ in order to filter out clutter. This implies that the hanning window spectrum main lobe must be situated inside $S_{d}$. It is then important to notice that the main lobe width of a one-dimensional (1-D) hanning window spectrum in normalized frequency ${ }^{3}$ is $4 / N_{h}$ with $N_{h}$ as the window samples number. Thus, the real filter stopband width depends on the hanning window samples numbers, i.e., $M$ and $N$. The bigger the filter coefficient numbers $M$ and $N$, the narrower the real filter stopband. Indeed, the real filter stopband should be as close as possible to the ideal filter stopband, defined by $S_{d}$. In order to do so, the main lobe width of the hanning window along $\nu_{\mathrm{xn}}$, denoted as $L_{x}$, must be taken less than $2 \alpha_{x}$, while the main lobe width of the hanning window along $\nu_{n}$, which is denoted as $L$, must be taken less than $\nu_{s}$. In normalized frequency, $M$ and $N$ should be chosen as follows:

$$
\begin{aligned}
L_{x} & =\frac{4}{M}<\frac{2 \alpha_{x}}{\nu_{\mathrm{xs}}} \Longrightarrow M>\frac{2 \nu_{\mathrm{xs}}}{\alpha_{x}} \\
L & =\frac{4}{N}<\frac{\nu_{s}}{\nu_{s}} \Longrightarrow N>4 .
\end{aligned}
$$

Moreover, the numbers of filter coefficients $M$ and $N$ should be chosen odd for the filter to be symmetrical with respect to the origin. Thus, $N$ can be taken as 5 , whereas $M$ is the closest odd number greater than $2 \nu_{\mathrm{xs}} / \alpha_{x}$. Knowing $x_{1}$ and the space sampling frequency $\nu_{\mathrm{xs}}$, the filter order can be automatically computed due to (32) and (37). As an example, the magnitude spectra of the ideal and real filters designed to filter out clutter for the Bscan of Fig. 2 are represented in Figs. 17 and 18. The filter parameters are $x_{1}=1 \mathrm{~m}$ and $\nu_{\mathrm{xs}}=50 \mathrm{~m}^{-1}$. Hence, the values $M=51$ and $N=5$ can be taken for the filter order.

\footnotetext{
${ }^{3}$ The frequencies, denoted as $f$, are normalized with respect to the sampling frequency $f_{s}\left(f /\left(f_{s}\right)\right.$ so that $f \in[-1 / 2,1 / 2]$.
}

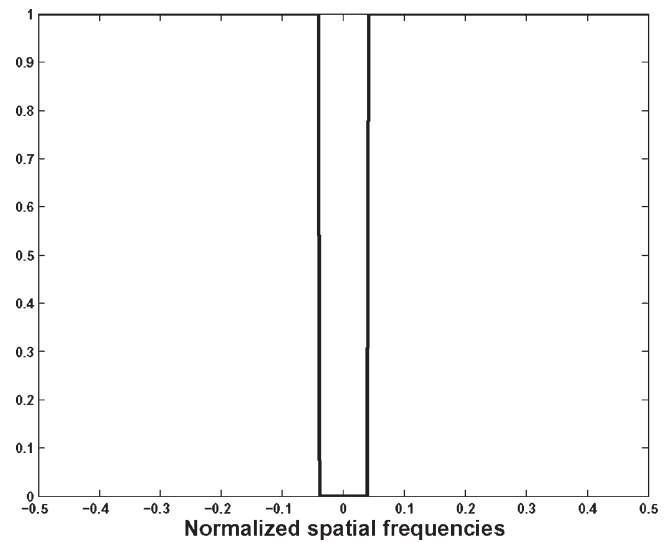

Fig. 17. Magnitude spectrum of the ideal filter for $\nu=0$.

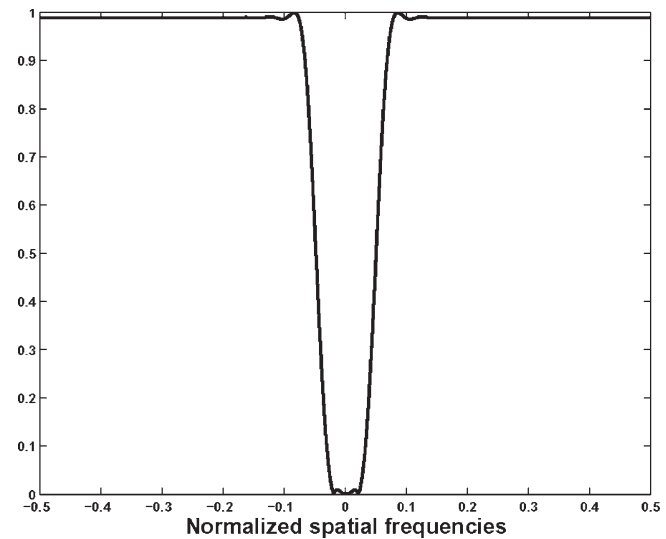

Fig. 18. Magnitude spectrum of the real filter for $\nu=0$.

\section{Simulations}

The digital high-pass filter specified previously is now applied to simulated and real data in order to test its abilities in terms of clutter removal.

\section{A. Simulated Data}

This new filter is applied to the simulated data depicted in Fig. 19. These data are made of a horizontal band that models the clutter and of three line segments that model a landmine signature buried at a shallow depth, as described in Section III-B. The horizontal band length $x_{1}$ is $1 \mathrm{~m}$. The length of the three line segments is $10 \mathrm{~cm}$, and the slopes of the two line segments modeling the hyperbola branches are 1 and -1 , respectively. The clutter magnitude is taken three times greater than the landmine signal magnitude. The space sampling frequency is $\nu_{\mathrm{xs}}=50 \mathrm{~m}^{-1}$. Thus, the ideal digital high-pass filter $H_{d}\left(\nu_{\mathrm{xn}}, \nu_{n}\right)$ is defined as

$$
\begin{cases}H_{d}\left(\nu_{\mathrm{xn}}, \nu_{n}\right)=0, & \left(\nu_{\mathrm{xn}}, \nu_{n}\right) \in S_{d} \\ H_{d}\left(\nu_{\mathrm{xn}}, \nu_{n}\right)=1, & \left(\nu_{\mathrm{xn}}, \nu_{n}\right) \notin S_{d}\end{cases}
$$

with $S_{d}=\left\{\left(\nu_{\mathrm{xn}}, \nu_{n}\right)\right.$ such that $\left|\nu_{\mathrm{xn}}\right| \in[0,1.98]$ and $\left|\nu_{n}\right| \in$ $[0,25]\}$. The values $N=5$ and $M=51$ are chosen for the filter order. The filtered data are shown in Fig. 20. The clutter has been significantly reduced, with the magnitude of the landmine signature being greater now than that of the clutter. 


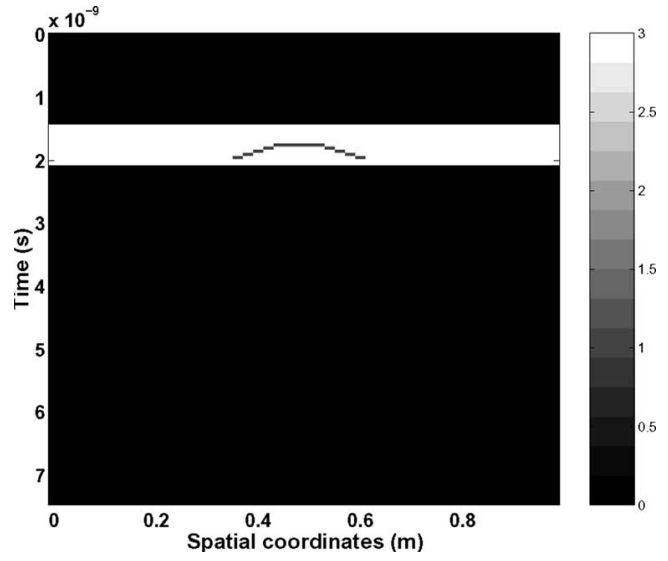

Fig. 19. Geometrical model of a Bscan.

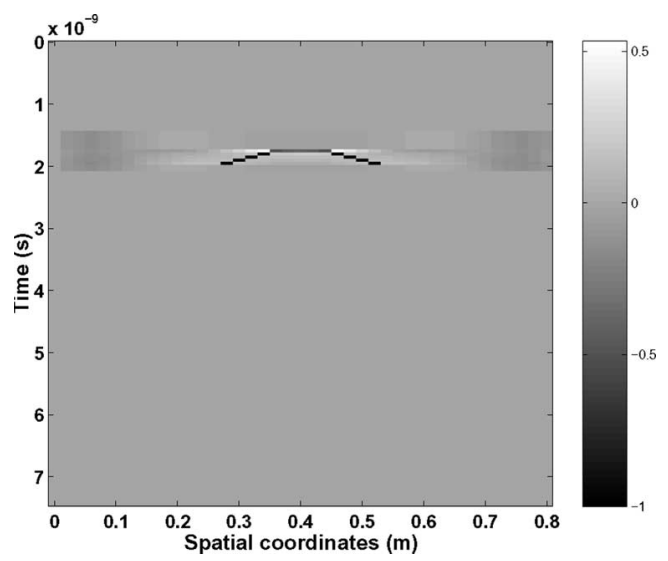

Fig. 20. Geometrical model of the Bscan shown in Fig. 19 with normalized magnitude (after digital filtering).

Furthermore, the modeled landmine signature has not been distorted by the filtering.

Of course, this result was attempted because the filter was designed from the frequency analysis of such data (Section III-B). More realistic simulated data, where landmine signatures appear as hyperbolas, are now considered in order to see if the geometrical model used for the frequency analysis of the hyperbola spectrum is good (Fig. 21). The split-step 2-D method presented in [20] was used to generate this Bscan. An EM pulse, which is modeled by a Gaussian function, is sent at a height of $12 \mathrm{~cm}$ above a homogeneous ground in which two objects have been placed. The central frequency of the pulse spectrum is $900 \mathrm{MHz}$. To apply the split-step method, the relative dielectric permittivity $\left(\varepsilon_{r}\right)$ and the quality factor $(Q)$ of the soil and objects must be known. The coupling effects between antennas that arise for bistatic GPR are not taken into account. The simulation parameters are given in Table I.

In order to design the filter, the parameters $x_{1}, M$, and $N$ should be determined. The horizontal band length $x_{1}$ is $3.78 \mathrm{~m}$, and the space sampling frequency $\nu_{\mathrm{xs}}$ is $33.3 \mathrm{~m}^{-1}$. The values $N=5$ and $M=129$ are taken for the filter order. The stopband of the digital filter can be found as $S_{d}=$ $\left\{\left(\nu_{\mathrm{xn}}, \nu_{n}\right)\right.$ such that $\left|\nu_{\mathrm{xn}}\right| \in[0,0.52]$ and $\left.\left|\nu_{n}\right| \in[0,16.6]\right\}$. Fig. 22 shows the simulated Bscan after filtering. It can be seen that the clutter has been entirely removed and that the buried objects appear as hyperbolas in the filtered Bscan.

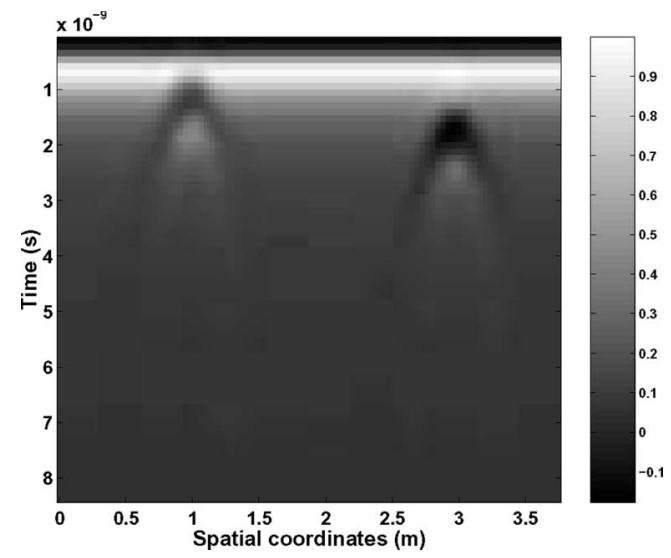

Fig. 21. Simulated Bscan.

TABLE I

Simulation PARAMETERS FOR THE BSCAN IN FIg. 21

\begin{tabular}{|c|c|c|c}
\hline & Object 1 & Object 2 & soil \\
\hline$\left(\varepsilon_{r}, Q\right)$ & $(3,13.5)$ & $(3,13.5)$ & $(10,30)$ \\
horizontal positions & $\{0.84,0.96\}$ & $\{2.3,2.45\}$ & - \\
depth & $2 \mathrm{~cm}$ & $5 \mathrm{~cm}$ & - \\
\hline
\end{tabular}

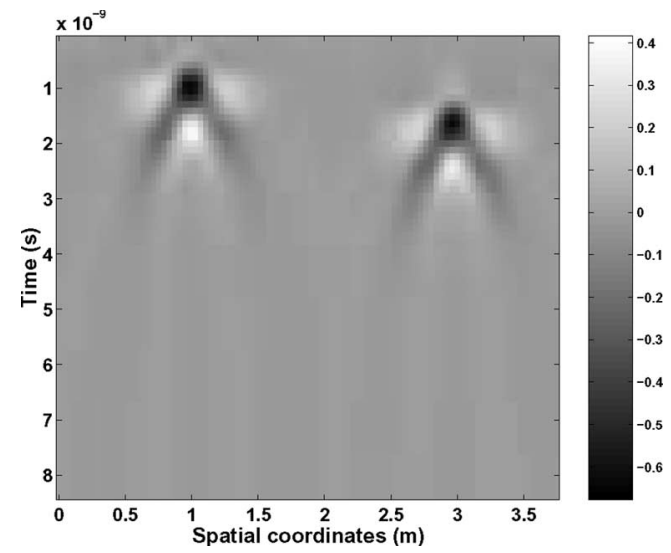

Fig. 22. Simulated Bscan in Fig. 21 with normalized magnitude (after digital filtering).

On the Bscan data of Figs. 19 and 21, clutter appears as a perfectly horizontal band. This implies that the air-ground interface is flat, which might not be realistic in the case of real GPR measurements. The last simulated data are used to study the case of a rough air-ground interface. The Bscan of Fig. 23 was created due to the split-step method. An EM pulse, modeled by a Gaussian function, is sent above a homogeneous ground in which two objects have been placed. The soil surface is a random variable which is centered in $z=0$ and whose variance is $5 \mathrm{~cm}$. The simulation parameters for these data are given in Table II.

The filter parameters are the same as that for the Bscan of Fig. 21; the filtered Bscan is represented in Fig. 24. It can be seen that most of the clutter has been canceled by the filtering but there are some clutter residues. Indeed, in an image, the low frequencies correspond to low-intensity variations (uniform zones), and the high frequencies correspond to fast variations (outlines). Hence, in a general framework, a high-pass filter 


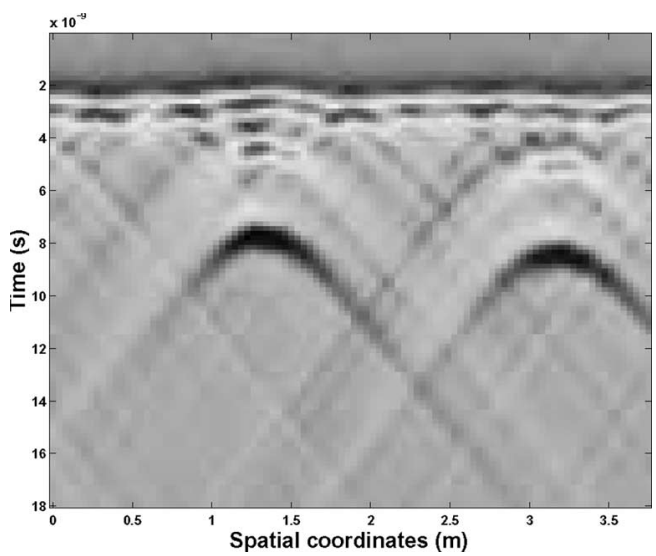

Fig. 23. Simulated Bscan

TABLE II

SIMULATION PARAMETERS FOR THE BSCAN IN FIG. 23

\begin{tabular}{|c|c|c|c|}
\hline & Object 1 & Object 2 & soil \\
\hline$\left(\varepsilon_{r}, Q\right)$ & $(10,5000)$ & $(10,5000)$ & $(3,60)$ \\
horizontal positions & $\{1.2,1.5\}$ & $\{2.9,3.2\}$ & - \\
depth & $5 \mathrm{~cm}$ & $10 \mathrm{~cm}$ & $\pm 5 \mathrm{~cm}$ \\
\hline
\end{tabular}

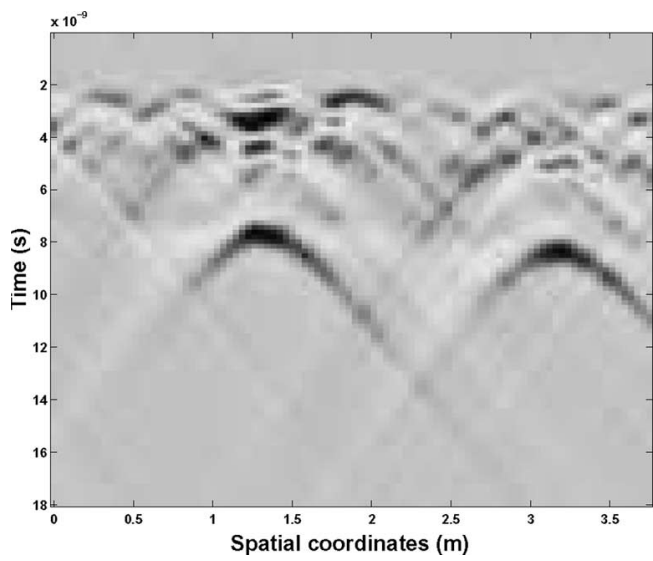

Fig. 24. Simulated Bscan in Fig. 23 with normalized magnitude (after digital filtering).

tends to increase the outlines and cancel the uniform zones. For clutter bands that are not perfectly horizontal, the outlines of clutter high-variation zones are at the origin of clutter residues (Fig. 24). However, the landmine responses are well represented after digital filtering, and their magnitude is greater than the one coming from clutter residues. The designed filter is therefore robust because it cancels most clutter even for roughsurface soils. This filter is therefore adapted to the Bscan data, and it can now be tested on real data.

\section{B. Real Data}

The MACADAM campaign real Bscan data, presented in Section II, are used to test our digital filter. The three real Bscans are selected such that they represent the recordings of a GPR above different types of soils, where several types of landmines are buried at different depths. For these Bscans, the filter parameters are the same, that is, $x_{1}=1 \mathrm{~m}$ and

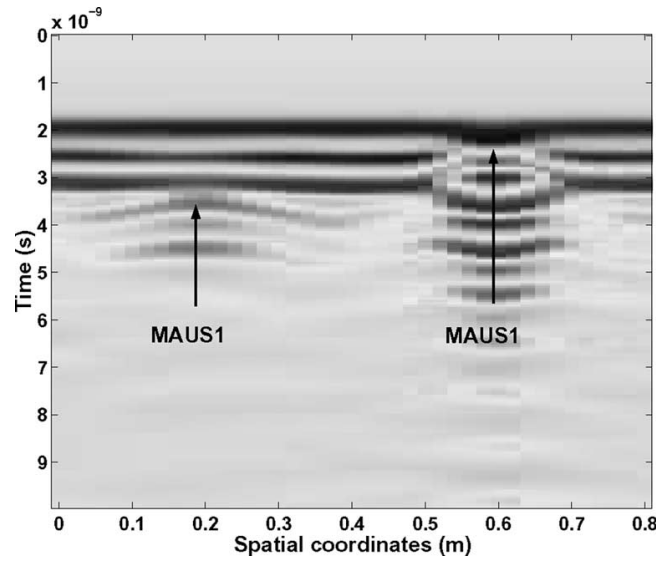

Fig. 25. Bscan recorded above an agricultural soil covered by grass.

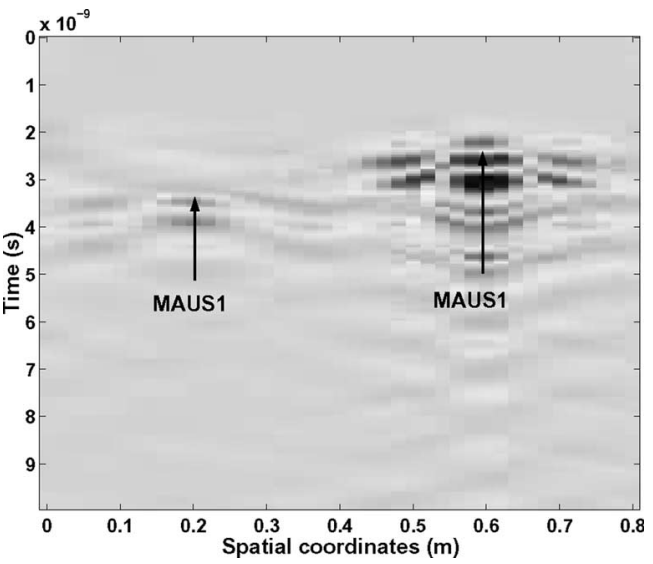

Fig. 26. Bscan in Fig. 25 after digital filtering $(N=5$ and $M=51)$

$\nu_{\mathrm{xs}}=50 \mathrm{~m}^{-1}$. The values $M=51$ and $N=5$ can be taken for the filter order. The stopband of the digital filter is $S_{d}=$ $\left\{\left(\nu_{\mathrm{xn}}, \nu_{n}\right)\right.$ such that $\left|\nu_{\mathrm{xn}}\right| \in[0,1.98]$ and $\left.\left|\nu_{n}\right| \in[0,25]\right\}$.

Consider the Bscan displayed in Fig. 25. In this image, two landmine responses of MAUS1 type (metallic content) have been recorded. One is coming from a landmine buried at $5 \mathrm{~cm}$, and the other is coming from a landmine laid down the ground at respective horizontal positions $x=0.2$ and $x=0.6$. The ground is made of clay covered by grass. The result is depicted in Fig. 26. The three bands representing the clutter are well filtered, and the landmine responses are not significantly distorted by the filtering.

The proposed filter is now tested on the Bscan shown in Fig. 27. Two VSMK2 landmine responses have been recorded on this Bscan, but they are hardly visible because their responses overlap with the one from the clutter. As shown in Fig. 28, after digital filtering, the three horizontal bands have been entirely filtered, and the landmine signatures appear as hyperbolas in the filtered Bscan.

Finally, the Bscan depicted in Fig. 29 is filtered. In this record, there are MAUS1 landmines buried at position $x=0.2$ at $10 \mathrm{~cm}$ and at position $x=0.6$ just below the air-ground interface. As shown in Fig. 30, the clutter bands have almost been entirely filtered, but there are some residues. Indeed, our filter has been constructed for clutter whose bands are almost horizontal. Now, as shown in Fig. 29, the clutter bands are not horizontal in the central part of the Bscan. Clutter residues 


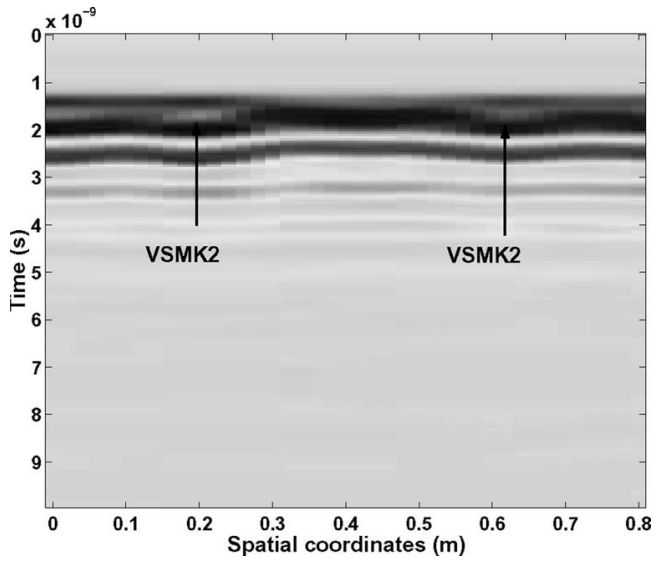

Fig. 27. Bscan recorded above an agricultural soil.

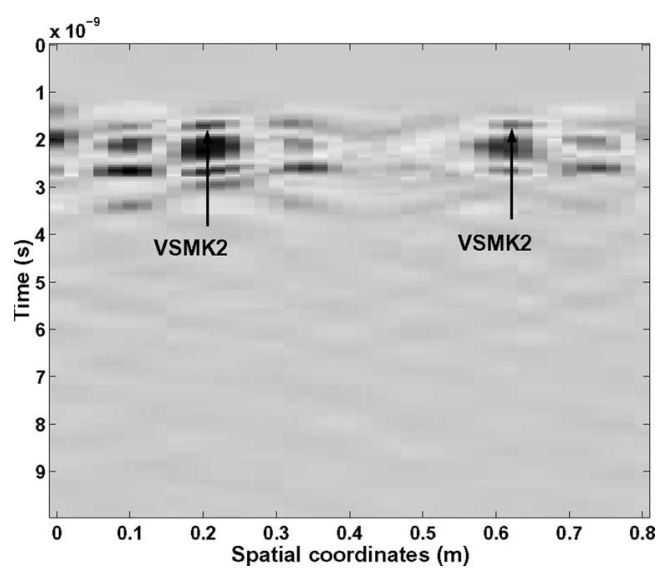

Fig. 28. Bscan in Fig. 27 after digital filtering $(N=5$ and $M=51)$.

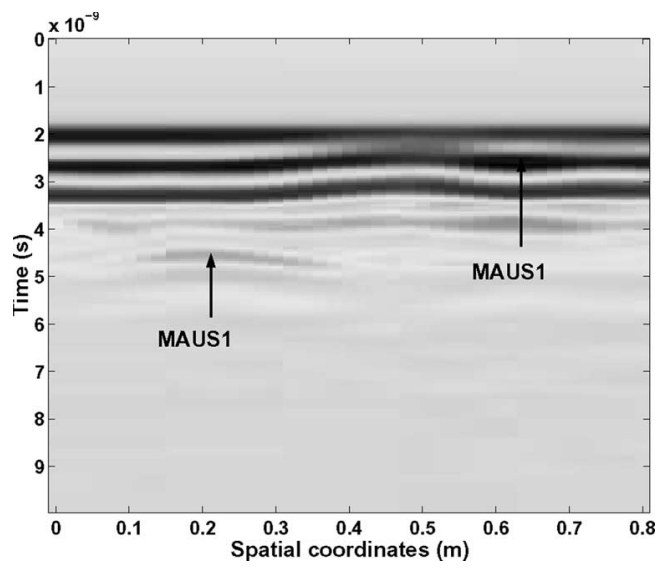

Fig. 29. Bscan recorded above an agricultural soil.

come from the outlines of the clutter bands in this zone. However, the two landmines are correctly represented on the Bscan after digital filtering, and their energy is greater than the one coming from clutter residues.

\section{Discussion}

The digital filtering approach for clutter reduction is effective for the real data set. These data represent different scenarios because the landmines are buried at different depths $(1-10 \mathrm{~cm})$ or laid down the ground. For all cases, the clutter is significantly

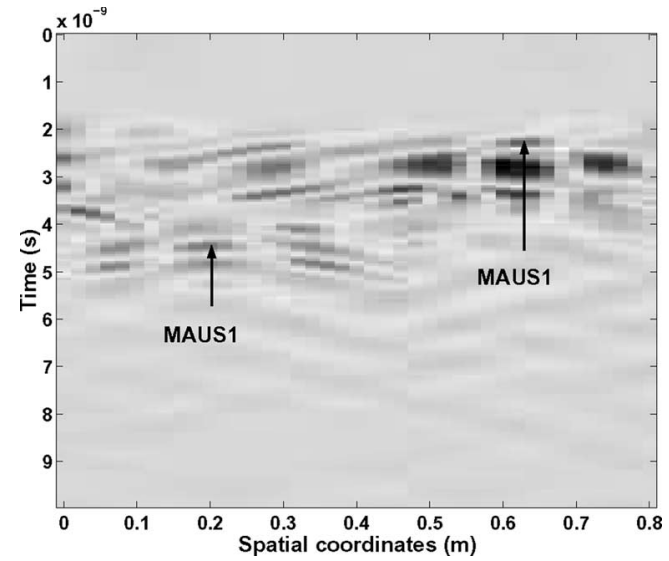

Fig. 30. Bscan in Fig. 29 after digital filtering ( $N=5$ and $M=51)$.

reduced, whereas the landmine signatures are protected, even for landmines whose responses are hidden by clutter. Thus, the proposed filter is well adapted to Bscan data. Its main drawback is that to get a perfect clutter reduction on Bscan data, the clutter should be made of horizontal bands, implying that the air-ground interface should be flat. If not, there might be some clutter residues after digital filtering. These residues come from the outlines of clutter bands high-variation zones. However, their magnitudes are attenuated by the filtering and are often lower than the one of landmine responses. Thus, the designed filter is robust and can be used to cancel clutter even for a rough air-ground interface. It can then be noticed that this digital filter has been successfully used as a preprocessing step of an abrupt change detection algorithm for the localization of landmine responses in Bscan records [18].

\section{Vi. Performances Analysis of the Clutter Removal Method}

A comparison with the commonly used CCRA is made in order to evaluate the performances of our method. Clutter reduction by CCRA is achieved by subtracting from each Ascan an averaged value of the $N_{w}$ previous Ascans [1], i.e.,

$$
x_{f}(n)=x(n)-\frac{1}{N_{w}} \sum_{i=1}^{N_{w}} x(n-i)
$$

where $x(n)$ is the vector that contains the Ascan samples recorded at position $n$ and $x_{f}(n)$ is the processed Ascan. The $z$ transform $X_{f}(z)$ of the processed Ascan $x_{f}(n)$ is defined as

$$
X_{f}(z)=X(z)\left(1-\frac{1}{N_{w}} \sum_{i=1}^{N_{w}} z^{-i}\right)
$$

where $z$ is a complex variable and $X(z)$ is the $z$ transform of $x(n)$. Hence, the CCRA realizes a clutter processing in the time domain by a digital filter whose transfer function $H_{\mathrm{ca}}(z)$ is defined as

$$
H_{\mathrm{ca}}(z)=\sum_{i=0}^{N_{w}} a_{i} z^{-i}
$$




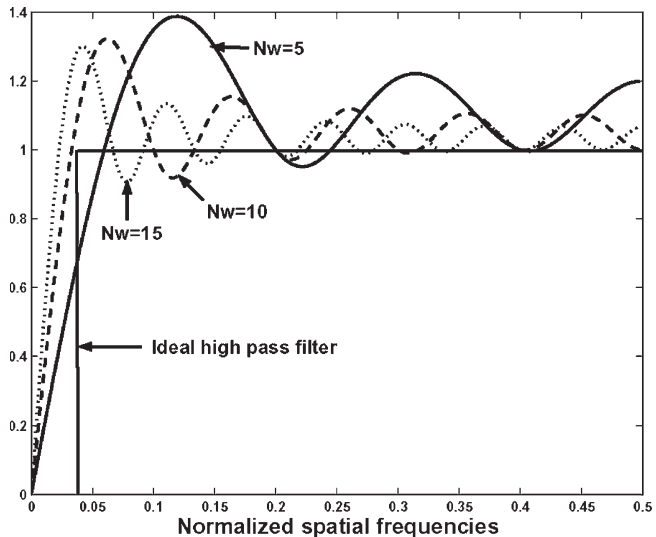

Fig. 31. Frequency responses of a CCRA filter for $N_{w}=5,10$, and 15 .

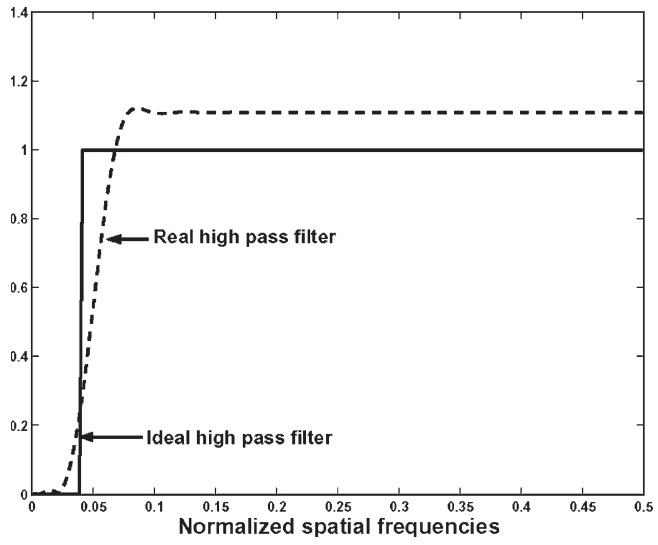

Fig. 32. Frequency responses of the real and ideal high-pass filter.

TABLE III

$R_{c}, R_{t}$, AND $R_{b}$ FOR THE DIFFERENT FILTERS

\begin{tabular}{|c|c|c|c|c}
\hline & Adapted filter & CCRA filter & CCRA filter & CCRA filter \\
\hline Order & $M=51, N=5$ & $N_{w}=5$ & $N_{w}=10$ & $N_{w}=15$ \\
\hline$R_{c}$ & $96 \%$ & $91,7 \%$ & $85 \%$ & $78.3 \%$ \\
\hline$R_{t}$ & $40 \%$ & $70 \%$ & $43.6 \%$ & $31 \%$ \\
\hline$R_{b}$ & $33.1 \%$ & $62.4 \%$ & $36.6 \%$ & $25.6 \%$ \\
\hline
\end{tabular}

where the filter coefficients $a_{i}$ are given such that $a_{0}=1$ and $a_{i}=-1 / N_{w}$ for $i=1, \ldots, N_{w}$. Thus, the filter coefficients depend only on the filter order $N_{w}$. The frequency responses of a CCRA filter for different values of $N_{w}$ are represented in Fig. 31. It can be seen that the settling time of these CCRA filters is quite long in comparison with the settling time of our filter and that their peak overshoots are greater (see Fig. 32). The frequency responses of CCRA filters are more distant from the ideal high-pass filter frequency response, defined by $H_{d}\left(\nu_{\mathrm{xn}}, \nu_{n}\right)$, than our filter frequency response. This can be explained by the fact that the CCRA filter coefficients are not optimized with respect to the clutter and landmine response approximated spectra.

In order to evaluate the performance of our clutter removal method against the CCRA, two criteria are used. The first criterion is the percentage measurement of the clutter power, denoted as $R_{c}$, that is filtered out for the two methods in order to see which method is the most efficient in terms of clutter removal. The second criterion is the percentage of the landmine

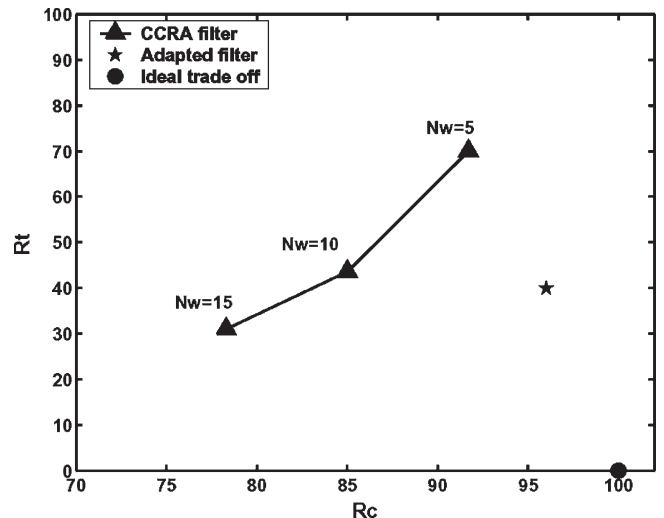

Fig. 33. $R_{t}$ is plotted against $R_{c}$ for the two filter types.

signal power that is filtered out for the two methods in order to see which method brings the least distortions to the landmine responses.

In Section III, the signatures of landmines buried at shallow depths have been modeled by three line segments whose magnitude spectra are defined by (25), (27), and (28), whereas the clutter magnitude spectrum is defined by (4). Hence, the percentage of distortions brought by the filtering on the hyperbola top $R_{t}$ can be defined as

$$
R_{t}=\frac{\int_{-\infty}^{\infty}\left|\operatorname{sinc}\left(\pi \Delta_{t} \nu_{x}\right)\right|^{2}|1-H|^{2} d \nu_{x}}{\int_{-\infty}^{\infty}\left|\operatorname{sinc}\left(\pi \Delta_{t} \nu_{x}\right)\right|^{2} d \nu_{x}} \times 100
$$

where $H$ is the transfer function of the filter used to reduce clutter. The maximum percentage of distortions brought by the filtering on the hyperbola branches $R_{b}$ is defined as

$$
R_{b}=\frac{\int_{-\infty}^{\infty}\left|\operatorname{sinc}\left(\pi \Delta_{b} \nu_{x}\right)\right|^{2}|1-H|^{2} d \nu_{x}}{\int_{-\infty}^{\infty}\left|\operatorname{sinc}\left(\pi \Delta_{b} \nu_{x}\right)\right|^{2} d \nu_{x}} \times 100 .
$$

Finally, the percentage of the clutter power that is filtered out along $\nu_{x}$ is defined as

$$
R_{c}=\frac{\int_{-\infty}^{\infty}\left|\operatorname{sinc}\left(\pi x_{1} \nu_{x}\right)\right|^{2}|1-H|^{2} d \nu_{x}}{\int_{-\infty}^{\infty}\left|\operatorname{sinc}\left(\pi x_{1} \nu_{x}\right)\right|^{2} d \nu_{x}} \times 100
$$

From the observations of our real database Bscans, the parameters $x_{1}, \Delta_{t}$, and $\Delta_{b}$ can be chosen as follows: $x_{1}=1 \mathrm{~m}$, $\Delta_{t}=10 \mathrm{~cm}$, and $\Delta_{b}=8 \mathrm{~cm}$. Table III gives the values of $R_{c}, R_{t}$, and $R_{b}$ for our filter and for CCRA filters. The best filter is the one that offers the best tradeoff between clutter reduction and landmine signatures protection. $R_{t}$ and $R_{b}$ are plotted against $R_{c}$ in Figs. 33 and 34, respectively, in order to see which filter is the closest to the ideal tradeoff $\left(R_{c}=100 \%\right.$ and $R_{b}=R_{t}=0 \%$ ). It can be seen that our clutter removal method is better than the CCRA because it offers the best tradeoff. To illustrate this, the Bscan of Fig. 29 is processed independently by two CCRA filters whose orders are $N_{w}=5$ and $N_{w}=10$, respectively. Fig. 35 shows that, for $N_{w}=5$, the clutter is significantly reduced but the signature of the MAUS1 landmine buried at $10 \mathrm{~cm}$ is cut into two parts. This can result in the detection of two buried objects instead of one, which leads to the increase of the false alarm rate. For $N_{w}=10$, it is shown in Fig. 36 that landmine signatures are less distorted, however 


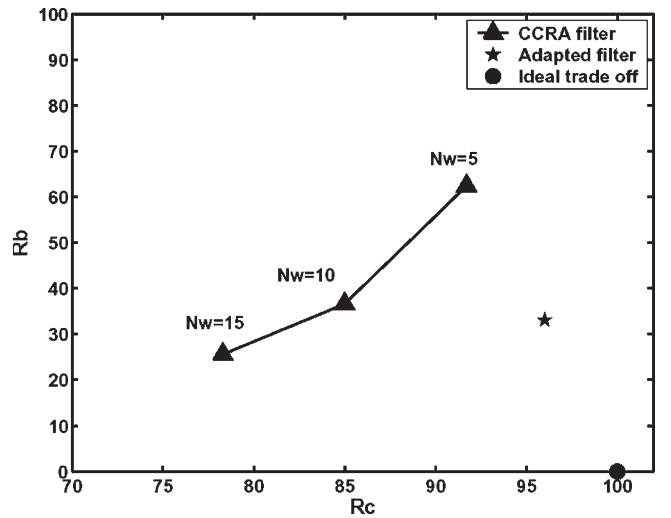

Fig. 34. $R_{b}$ is plotted against $R_{c}$ for the two filter types.

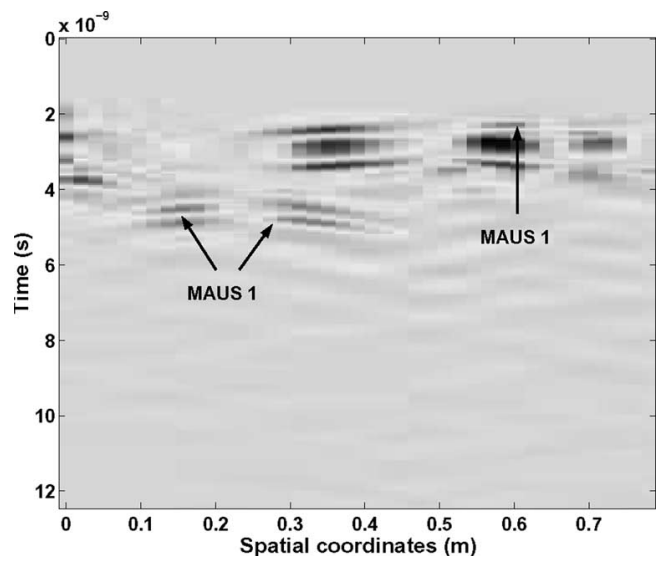

Fig. 35. Bscan in Fig. 29 after clutter removal by the CCRA filter $\left(N_{w}=5\right)$.

their magnitudes are lower than that of the clutter residues. The preprocessing of this Bscan by our filter is better (see Fig. 30).

Finally, the performance of our filter and CCRA filters for clutter reduction is studied in terms of detection probability and false alarm probability with the help of receiver operating characteristic (ROC) curves. The landmine detection method used is the one proposed in [18]. This method consists of detecting all abrupt changes in the Bscan data along both time and spatial dimensions and in finding those coming from the responses of buried landmines. More precisely, the two steps of this method are as follows.

Step 1) Spatial abrupt changes are searched in order to detect the possible horizontal landmines position. Clutter reduction is not necessary because clutter is almost constant along the horizontal axis of the real Bscans.

Step 2) The time abrupt changes are searched in order to detect the buried-object response times. Clutter has to be removed beforehand in order to avoid detecting clutter bands instead of real landmines.

Here, we are only interested in Step 2) of the landminedetection method because clutter reduction is used as a preprocessing step. In order to build an ROC curve for each filter, a set of 75 real Bscan data collected by a bench arc, as described in Section II, is used. The laying configuration of the landmines is shown in Fig. 3. Five MAUS1 landmines and one AUPS landmine have been buried at different depths in an agricultural soil without any other objects, such as twigs or rocks. For

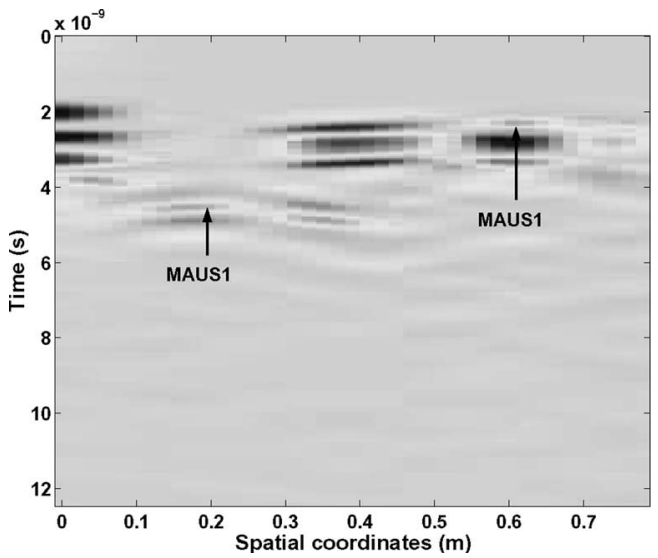

Fig. 36. Bscan in Fig. 29 after clutter removal by the CCRA filter $\left(N_{w}=10\right)$

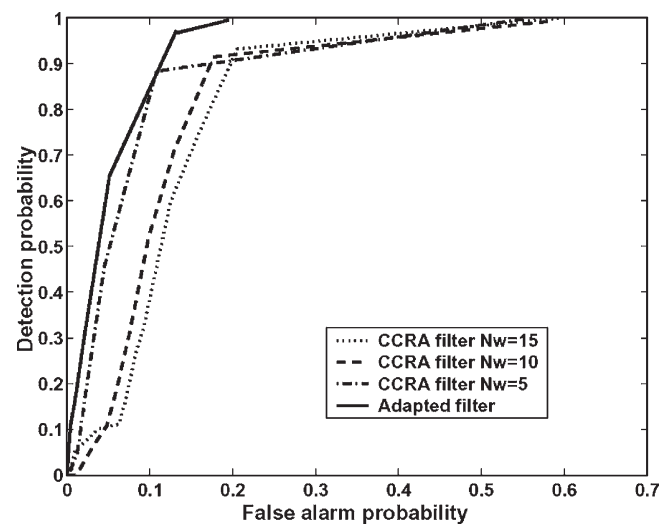

Fig. 37. ROC curves for the detection of landmine response time [Step 2)] when the Bscan data are preprocessed by the adapted filter and the CCRA filters.

each Bscan, we apply Step 2) of the detection method. The Bscan data are first preprocessed by one of the filters, and then the abrupt change detection algorithm is applied along the time direction. The algorithm parameters are $m_{1}=m_{2}=5$, $\sigma=10$, and $\nu=0.5$ [18]. A buried object is characterized by two near-abrupt changes in the time direction which are relative to the response times of the top and bottom of the object. Hence, for each detected response time, if it corresponds to a theoretical response time of a landmine, the detection is true; otherwise, it is considered a false alarm. Then, the probability of detection and the probability of false alarm are computed for different values of the detection threshold $\eta_{t}\left(\eta_{t} \in[0,12]\right)$. The ROC curves for the different filters are plotted in Fig. 37. It can be seen that the detection-false alarm performance is better when the Bscans from the data set are preprocessed by our filter rather than by CCRA filters.

\section{CONCLUSION}

In this paper, a digital high-pass filter that is adapted to Bscan data has been proposed to reduce clutter. The different simulation results show the efficiency of such a filter to remove clutter while protecting the landmine signatures, even for landmines whose responses are hidden by clutter. The performances of our method were evaluated due to a comparison 
with the commonly used CCRA method, which shows that our method gives a better tradeoff in terms of clutter reduction and landmine signatures protection and a better detection performance when it is used as a preprocessing step for the landmine-detection method proposed in [18]. The only thing that should be checked before applying this filter to any Bscan data is that the width of the buried object in the Bscan image is small in comparison with the image width. Moreover, the implementation of such a filter is simple, and its computational cost is low.

\section{ACKNOWLEDGMENT}

The authors would like to thank the reviewers for their comments and suggestions in improving the quality of this paper. The authors are grateful to the Foundation Norbert Ségard for its financial contribution.

\section{REFERENCES}

[1] D. J. Daniels, Ground-Penetrating Radar, 2nd ed. London, U.K.: IEE, 2004.

[2] L. Chety, "Handheld mine detection test platform-SDD, IDD," Thomson-CSF Missile Electronics, Paris, France, Tech. Rep. TME/DM/ 1998/4330/LC/BDH, Dec. 1998.

[3] N. Milisavljevic, "Analyse et Fusion par la Théorie des Fonctions de Croyances de Données Multi-sensorielles pour la Détection de Mines Anti-personnelles," Ph.D. dissertation, Telecom, Paris, France, 2001.

[4] A. Van der Merwe and I. J. Gutpa, "A novel signal processing technique for clutter reduction in GPR measurements of small, shallow land mines," IEEE Trans. Geosci. Remote Sens., vol. 38, no. 6, pp. 2627-2637, Nov. 2000

[5] I. J. Gupta, A. Van der Merwe, and C.-C. Chen, "Extraction of complex resonances associated with buried targets," in Proc. SPIE-Detection Remediation Technol. Mines Mine-Like Targets III, Jul. 1997, vol. 3392, pp. 568-580.

[6] D. D. Carevic, M. Craig, and I. Chant, "Modelling GPR echoes from land mines using linear combination of exponentially damped sinusoids," in Proc. SPIE-Detection Remediation Technol. Mines Mine-Like Targets II, Sep. 1998, vol. 3079, pp. 1022-1032.

[7] A. Farina and A. Protopa, "New results on linear prediction for clutter cancellation," IEEE Trans. Aerosp. Electron. Syst., vol. 24, no. 3, pp. 275-285, May 1998.

[8] K. Ho and P. D. Garder, "A linear prediction land mine detection algorithm for hand held ground penetrating radar," IEEE Trans. Geosci. Remote Sens., vol. 40, no. 6, pp. 1374-1384, Jun. 2002.

[9] B. Cagnoly and T. J. Ulrych, "Singular value decomposition and wavy reflections in ground-penetrating radar images of base surge deposits," J. Appl. Geophys., vol. 48, no. 3, pp. 175-182, Oct. 2001.

[10] B. Karlsen, B. D. Sorensen, J. Larsen, and K. B. Jakobson, "Independent component analysis for clutter reduction in ground penetrating radar data," in Proc. SPIE-Aerosense, 2002, vol. 4742, pp. 378-389.

[11] — , "GPR detection of buried symmetrically shaped mine-like objects using selective independent component analysis," in Proc. Detection Remediation Technol. Mines Mine-Like Targets-Aerosense, 2003, vol. 5089, pp. 375-386.

[12] S. Tjora, E. Eide, and L. Lundheim, "Evaluation of methods for ground bounce removal in GPR utility mapping," in Proc. 10th Int. Conf. Ground Penetrating Radar, Delft, The Netherlands, Jun. 2004, pp. 379-382.

[13] A. Zhao, Y. Jiang, W. Wang, and X. Jiaotong, "Exploring independent component analysis for GPR signal processing," in Proc. Progress Electromagn. Res. Symp., Hangzhou, China, Aug. 22-26, 2005, pp. 750-753.

[14] S. Ebihara, "Blind separation for estimation of near-surface interface by GPR with time-frequency distribution," IEICE Trans. Commun., vol. E86-B, no. 10, pp. 3071-3080, Oct. 2003.

[15] K. C. Ho, P. D. Gader, and J. N. Wilson, "Improving landmine detection using frequency domain features form ground penetrating radar," in Proc. Geosci. Remote Sens. Symp., 2004, vol. 3, pp. 1617-1620.

[16] P. D. Gader, J. M. Keller, and B. Nelson, "Recognition technology for the detection of buried landmines," IEEE Trans. Fuzzy Syst., vol. 9, no. 1, pp. 31-43, Feb. 2001.
[17] Q. Zhu and L. M. Collins, "Application of feature extraction methods for landmine detection using the Wichmann/Niitek ground penetrating radar," IEEE Trans. Geosci. Remote Sens., vol. 43, no. 1, pp. 81-85, Jan. 2005

[18] D. Potin, P. Vanheeghe, E. Duflos, and M. Davy, "An abrupt change detection algorithm for buried landmines localization," IEEE Trans. Geosci. Remote Sens., vol. 44, no. 2, pp. 260-272, Feb. 2006.

[19] A. V. Oppenheim and D. W. Shafer, Digital Signal Processing. Englewood Cliffs, NJ: Prentice-Hall, 1975.

[20] A. Bitri and G. Grandjean, "Frequency-wavenumber modeling and migration of 2D GPR data in moderately heterogenous dispersive media," Geophysics, vol. 46, no. 3, pp. 287-301, 1998.

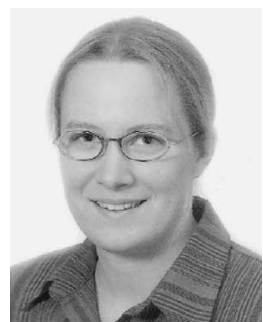

Delphine Potin was born in Liévin, France, on September 12, 1979. She received the B.S. degree in engineering from the Institut Supérieur d'Electronique du Nord, Lille, France, and the M.Sc. degree in advanced control and systems engineering from the Institute of Science and Technology, University of Manchester, Manchester, U.K., both in 2002. She is currently working toward the Ph.D. degree at the French CNRS laboratory "Laboratoire d'Automatique, Genie Informatique et Signal" (LAGIS UMR CNRS).

Her current research interests include personnel landmine detection.

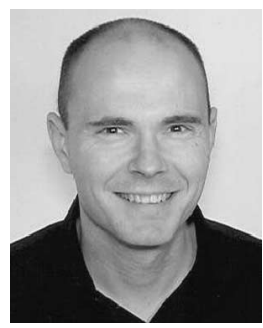

Emmanuel Duflos (M'00) was born in Amiens, France, on June 20, 1968. He received the B.S. degree in engineering from the Institut Supérieur d'Electronique du Nord (ISEN), Lille, France, in 1991, the Diploma of Advanced Study in automatic control and signal processing from the University of Paris XI, Orsay, France, in 1992, the Ph.D. degree in engineering sciences from the Université de Toulon et du Var, La Garde, France, in 1995, and the Habilitation à Diriger des Recherches from the University of Lille I, Lille, in 2003.

$\mathrm{He}$ is currently a Professor with the Ecole Centrale de Lille, Laboratoire d'Automatique, Génie Informatique et Signal, Villeneuve d'Ascq Cedex, France, after spending eight years at ISEN. He is a coauthor, together with Prof. Philippe Vanheeghe, of several papers about guidance law modeling, multisensor management systems with application to radar sensor management, and personnel landmine detection. His current research interests include multisensor systems, ranging from signal analysis for data fusion to multisensor management in moving multitarget environments. He is a member of the French CNRS laboratory "Laboratoire d'Automatique, Genie Informatique et Signal" (LAGIS UMR CNRS).

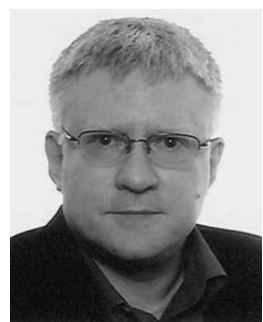

Philippe Vanheeghe (M'92-SM'98) was born in France on July 20, 1956. He received the M.S. degree in data processing, the Diploma of Advanced Study in data processing, the Ph.D. degree, and the Habilitation à Diriger des Recherches from the University of Lille, Lille, France, in 1981, 1982, 1984, and 1996, respectively.

He was an Assistant Professor with the Institut Supérieur d'Electronique du Nord, Lille, and was promoted to Head of the Signals and Systems Department in 1990. He is currently a Professor with the Ecole Centrale de Lille, Lille. He is with the Head of the French CNRS laboratory "Laboratoire d'Automatique, Génie Informatique et Signal" (LAGIS UMR CNRS). He is a coauthor, together with Prof. Emmanuel Duflos, of several papers about guidance law modeling, multisensor management systems with application to radar sensor management, and personnel landmine detection. His research interests include multisensor management, signal processing, signals, and systems modeling.

Dr. Vanheeghe was a member of the International Program Committee of several international symposiums (IEEE, IMACS, etc.) and a Session Organizer for many international conferences. 